\title{
Network Oscillations in Rod-Degenerated Mouse Retinas
}

\author{
Jacob Menzler and Günther Zeck \\ Department of Systems and Computational Neuroscience, Max Planck Institute of Neurobiology, D-82152 Martinsried, Germany
}

In the mammalian retina, excitatory and inhibitory circuitries enable retinal ganglion cells (RGCs) to signal the occurrence of visual features to higher brain areas. This functionality disappears in certain diseases of retinal degeneration because of the progressive loss of photoreceptors. Recent work in a mouse model of retinal degeneration $(r d 1)$ found that, although some intraretinal circuitry is preserved and RGCs maintain characteristic physiological properties, they exhibit increased and aberrant rhythmic activity. Here, extracellular recordings were made to assess the degree of aberrant activity in adult $r d 1$ retinas and to investigate the mechanism underlying such behavior. A multi-transistor array with thousands of densely packed sensors allowed for simultaneous recordings of spiking activity in populations of RGCs and of local field potentials (LFPs). The majority of identified RGCs displayed rhythmic $(7-10 \mathrm{~Hz})$ but asynchronous activity. The spiking activity correlated with the LFPs, which reflect an average synchronized excitatory input to the RGCs. LFPs initiated from random positions and propagated across the retina. They disappeared when ionotrophic glutamate receptors or electrical synapses were blocked. They persisted in the presence of other pharmacological blockers, including TTX and inhibitory receptor antagonists. Our results suggest that excitation — transmitted laterally through a network of electrically coupled interneurons-leads to large-scale retinal network oscillations, reflected in the rhythmic spiking of most $r d 1$ RGCs. This result may explain forms of photopsias reported by blind patients, while the mechanism involved should be considered in future treatment strategies targeting the disease of retinitis pigmentosa.

\section{Introduction}

Enhanced neuronal oscillations and aberrant synchrony are characteristic features of disorders of the CNS, including the epilepsies and Parkinson's disease (for review, see Uhhaas and Singer, 2006). In these diseases, an imbalance between excitatory and inhibitory circuitry may cause enhanced excitability and subsequent aberrant synchronous behavior in neuronal populations. Gap junctions-intercellular channels that directly connect neighboring cells_-are thought to support such neuronal activity patterns (Rufer et al., 1996; Dudek et al., 1998; Carlen et al., 2000).

Recently, the rod-degenerated ( $r d 1)$ mouse retina, a model that mimics the human disease of retinitis pigmentosa (RP), has been reported to display some electrophysiological features reminiscent of pathological oscillatory discharge (Ye and Goo, 2007; Margolis et al., 2008; Stasheff, 2008). The degeneration of photoreceptors triggers changes in the retinal morphology in these mouse retinas (for review, see Marc et al., 2003), and in human RP patients (Kolb and Gouras, 1974; Fariss et al., 2000). Neurons postsynaptic to photoreceptors (horizontal and bipolar cells) retract their dendrites and develop aberrant axonal processes (Strettoi and Pignatelli, 2000; Strettoi et al., 2002), whereas amacrine cells and retinal ganglion cells (RGCs) maintain their morphology up to late degenerate stages (Stone et al., 1992; Santos et

\footnotetext{
Received Aug. 13, 2010; revised Dec. 6, 2010; accepted Dec. 10, 2010.

This work was supported by The Max Planck Society and by German Science Foundation Grant ZE 535/4-1 (G.Z.) We thank A. Lambacher for technical assistance with the spike-sorting algorithm and D. $\mathrm{Ng}$ for critical reading of this manuscript.

Correspondence should be addressed to Günther Zeck at the above address. E-mail: zeck@neuro.mpg.de or guenther.zeck@nmi.de.

DOI:10.1523/JNEUROSCI.4238-10.2011

Copyright $\odot 2011$ the authors $\quad 0270-6474 / 11 / 312280-12 \$ 15.00 / 0$
}

al., 1997; Humayun et al., 1999; Strettoi et al., 2003; Mazzoni et al., 2008). On a molecular level, functional remodeling of ionotrophic and metabotropic glutamate receptors in inner retinal neurons of adult $r d 1$ retinas has been reported (Chua et al., 2009); however, little is known about the expression patterns of inhibitory receptors or gap junctions (Fletcher, 2000).

In adult $r d 1$ retinas, projection neurons-the RGCs- develop increased spontaneous activity that is in many cases rhythmic and maintained for weeks after light-evoked responses disappear (Ye and Goo, 2007; Margolis et al., 2008; Stasheff, 2008; Ryu et al., 2010). Indirect evidence of rhythmic retinal activity was reported earlier in superior colliculus recordings of anesthetized $r d 1$ mice (Dräger and Hubel, 1978). It remained unclear whether the rhythmic activity occurred in a subset of RGCs, whether it is synchronized at the population level, and what are the mechanisms governing such activity.

Here, we investigate these questions by recording the electrical activity in RGC populations of adult $r d 1$ retinas $(\mathrm{C} 3 \mathrm{H}$ and FVB/N) using a high-density multi-transistor array (MTA) (Eversmann et al., 2003; Lambacher et al., 2004). The array comprises 16,384 electrodes packed in $1 \mathrm{~mm}^{2}$, thus allowing for very high spatial electrical imaging of the interfaced in vitro retina. Simultaneous recording from up to $100 \mathrm{RGCs}$ reveals that the majority of them spike at a rhythm of 7-10 Hz. This rhythmic spiking is driven by strong presynaptic input that causes spatially extended local field potentials (LFPs). Based on the LFP propagation velocity, the LFP sensitivity to gap junction blockers, and phase-shifted RGC spiking, we postulate that electrical synapses between retinal interneurons mediate the transmission of aberrant rhythmic activity. The presented results may explain some of the neuropathological findings in RP patients and may guide strategies to treat the disease. 


\section{Materials and Methods}

Animals. All experiments were performed in accordance to the animal use committee of the Max Planck Institutes. In this study, retinas from male $r d 1$ mice were investigated between postnatal day 35 (P35) and P70, when their retinas are no longer responsive to light. All experiments on rd1 retinas were first performed using the local colony of the FVB/ NCrlMPI strain that is homozygous for the $P d e 6 b^{r d l}$ mutation (Taketo et al., 1991). After the pharmacological protocols had been established, the experiments were repeated with $r d 1$ retinas from $\mathrm{C} 3 \mathrm{H} / \mathrm{HeNCrl}$ mice (Charles River) that are homozygous for the $P d e 6 b^{r d l}$ mutation. Control experiments were performed on $w t$ retinas from the local colony of C57BL/6NMPI male mice. To rule out possible interference from different genetic backgrounds, we additionally obtained $\mathrm{C} 3 \mathrm{H} w t$ male mice from F. Paquet-Durand (Tübingen, Germany). This mouse strain was initially created in the laboratory of S. Sanyal (Sanyal and Bal, 1973) by substituting the mutated Pde6b gene with the normal allele and has been maintained in various laboratories ever since. All animals were housed in temperature-regulated facilities on a $12 \mathrm{~h} \mathrm{light/dark} \mathrm{cycle} \mathrm{and} \mathrm{fed} \mathrm{ad} \mathrm{libitum.}$ All animals were dark adapted $(1 \mathrm{~h})$ before the retina preparation.

Preparation of the mouse retina and mounting on the multi-transistor sensor array. The preparation of the retina was performed under dim red illumination ( $640 \mathrm{~nm}$ LED; Roithner Lasertechnik) that also illuminated the room during the experiment. Mice were anesthetized with isoflurane (CP Pharma) and killed by cervical dislocation. Their eyes were removed, bathed in room temperature-oxygenated Ames' medium (Sigma-Aldrich; A1420; pH 7.4), and hemisected. Next, the lens and vitreous were removed from the eyecup, and finally the retina was gently peeled off the pigment epithelium. The retina was mounted ganglion cell side down on a poly-L-lysine (150 kDa molecular weight; Sigma-Aldrich)-coated MTA. The MTA itself was glued on a ceramic package (CPGA; Spectrum). The bond wires are shielded with a custom-made Perspex chamber with an inner area of $12 \mathrm{~mm}^{2}$. During the recording, retinal tissue on the MTA was continuously perfused with oxygenated Ames' medium $\left(33-36^{\circ} \mathrm{C}\right)$ at a rate of $7 \mathrm{ml} / \mathrm{min}$.

Electrical recording with multi-transistor arrays. The electrical response of the retina was measured using an array of $128 \times 128$ equally spaced sensor transistors covering an area of $1 \mathrm{~mm}^{2}$. For the default configuration, we measured every second column $(128 \times 64$ sensors $)$ with a sampling frequency of $12 \mathrm{kHz}$ for each sensor. In each experiment, sensor transistors were calibrated by applying an AC voltage (frequency, $70 \mathrm{~Hz}$; amplitude, $3 \mathrm{mV}$ peak-to-peak) to the bath electrode (Eversmann et al., 2003; Lambacher et al., 2004). The calibration voltage changes the electrical potential at the surface of the chip. The local change of electrical potential couples through the insulating electrolyte/chip interface to the gate of the sensor transistor and proportionally modulates the sourcedrain current therein. During the experiment, ion currents through excited retinal ganglion membranes change the local extracellular voltage with respect to the bath electrode. The potential at the chip surface couples through the insulating electrolyte/chip interface to the gate and proportionally modulates the source-drain current. The response of each sensor transistor is solely determined by the potential above the insulating $\mathrm{TiZrO}_{2}$ layer, averaged over the diameter $(6.3 \mu \mathrm{m})$ of the top contact. The insulating $\mathrm{TiZrO}_{2}$ layer had a thickness of $\sim 30 \mathrm{~nm}$. The chip readout pattern was optimized to avoid cross talk of transistor signals on the chip (Eversmann et al., 2003; Lambacher et al., 2004). During the recording, the columns of the sensor array were sequentially connected to 128 line amplifiers. After a settling time of $720 \mathrm{~ns}$, the output of these line amplifiers was multiplexed over another $640 \mathrm{~ns}$ into 16-output channels. The readout time of $128 \times 64$ sensor array was therefore approximately $(1.36 \times 64) \mu$ s. Within each sensor column, an 8:1 multiplexer selects 16 sensors (sensor spacing, $125 \mu \mathrm{m}$ ) that are read out within $\sim 640 / 8 \mathrm{~ns}$.

Identification of action potentials and assignment to the corresponding ganglion cell. The method for identifying action potentials and assignment to corresponding neurons has been described in a recent report (Lambacher et al., 2010). Briefly, the analysis is done in three steps: (1) identification of threshold crossings of a signal vector $V$ calculated from neighboring extracellular voltages, (2) assignment of threshold crossings to one action potential, and (3) assignment of action potentials to corresponding neurons.

For the identification of threshold crossings, we first apply a bandpass filter to the calibrated data $(0.1-3 \mathrm{kHz})$. As the sensor distance $(7.8 \mu \mathrm{m})$ is smaller than the ganglion cell soma size, each extracellular signal is picked up by more than one sensor. The duration of the somatic extracellular signal is longer than the time interval between consecutive data points. Therefore, for each recorded data point, the length of a signal vector $V$ is calculated as follows:

$$
V=\sqrt{\sum_{i=1}^{27} \frac{V_{i}^{2}}{\sigma_{i}^{2}}}
$$

with $V_{i}$ representing the signal amplitude of data point $i$ in neighborhood; $\sigma_{i}$, root mean square (rms) noise of transistor in neighborhood. The sum runs over a $3 \times 3 \times 3$ neighborhood (three sensor rows, three sensor columns, three time points) surrounding the data point under consideration. The data point itself is part of the neighborhood. If $V$ exceeds a threshold of 15 , the data point is saved and considered part of the extracellular waveform that represents the action potential. Assuming equal noise on each of the nine neighboring sensors and homogenous coupling on these sensors, the threshold value of 15 means that those extracellular voltages exceeding $15 / \sqrt{ } 27 \times \mathrm{rms}$ of the corresponding sensor are detected. This threshold value is close to that of previous studies using metal electrode arrays (Zeck and Masland, 2007; Stasheff, 2008 ) but slightly higher than the value of $11.7 / \sqrt{ } 27 \times$ rms used in the study by Lambacher et al. (2010).

In a second step, threshold crossings are combined to action potentials. All threshold crossings that are spatially adjacent at the same time point are merged into a "cluster." Next, we consider the spatial overlap of time-consecutive clusters. Two such clusters are part of the same action potential if they share at least one sensor. All clusters that belong to an action potential are combined and the data point (time stamp and sensor location) with the highest amplitude is chosen as a representative for the action potential.

Finally, action potentials are assigned to the corresponding ganglion cells. Action potentials recorded on one sensor may belong to different cells. We again take advantage of the high spatial sampling and align the centers of gravity of two action potentials in time. For each action potential we consider the extracellular voltages surrounding the center of gravity. The cross-correlation (CC) between the neighborhoods of action potentials $i$ and $j$ is calculated as follows:

$$
c_{i j}=\left(\sum_{k} V_{i, k} V_{j, k}\right) / N_{i} N_{j}
$$

with

$$
N_{i}=\sum_{l} V_{i, l}^{2}
$$

as the normalization factor for action potential $i$. The index $k$ ranges over all peaks that are common to both action potentials $i$ and $j$, whereas the index $l$ ranges over all voltages that constitute the action potential. The following analysis separates action potentials that originate from different neurons. For a number of $M$ overlapping action potentials, a symmetric matrix of cross-correlation values $c_{i j}(i, j=1: M)$ is obtained. This matrix is rearranged to minimize the $c_{i j}$ differences between adjacent rows. If all action potentials under consideration belong to one neuron, we obtain little variation within the correlation matrix. If action potentials from several neurons are compared, separate clusters are visible in the cross-correlation matrix. This matrix is then split and the action potentials within each cluster are assigned to a different neuron. The sorting of action potentials is comparable with the supervised algorithm used in a previous study, although, there, a different algorithm ( $k$-means clustering) was used (Zeck and Masland, 2007). The spike sorting and splitting is done off-line and semiautomated. The final spike trains are tested to obey a refractory period of at least $1 \mathrm{~ms}$. No action potentials 
with interspike intervals $<1 \mathrm{~ms}$ were assigned to one neuron using the described method.

Spike train analysis. Data were analyzed using custom software written in Matlab (The MathWorks). The average firing rate for each spontaneously active RGC was computed as the total number of spikes divided by the length of the recording period. The recording consists of 1-10 s concatenated segments of continuous voltage traces $(0.1-12 \mathrm{kHz}$ sampling frequency). The fundamental spiking frequencies were estimated from the autocorrelation functions.

CC functions were computed for cell pairs after spike trains had been assigned to particular cell. Normalized CCs were calculated using the Matlab routine xcorr. This routine calculates the dot product of two normalized vectors representing the RGCs spike trains. The spike trains were binned with either 4 or $0.4 \mathrm{~ms}$ time resolution. The correlation coefficient (Pearson's correlation) presented here represents the zeroth lag of the correlation function. It represents the percentage of spikes from one spike train that occurs in the same time bin in the correlated spike train and therefore represents an estimate of the coupling strength between two RGCs. The length of correlated spike trains ( $\sim 5 \mathrm{~min}$ in $4 \mathrm{~ms}$ time bins) sets the statistical significance of the correlation coefficient $<0.01$.

Local field potentials and propagation velocity. Extracellular voltage changes characterized by negative deflections ( $\sim 20 \mathrm{~ms}$ long) followed by slower repolarization $(\sim 100 \mathrm{~ms})$ have been reported as a slow-wave component in a recent $r d 1$ study using multielectrode arrays with large electrode distances (Ye and Goo, 2007; Ryu et al., 2010). Voltage modulations that occur in phase across neighboring sensors-but not across the whole sensor array $\left(1 \mathrm{~mm}^{2}\right)$-reflect spatially confined local field potentials. Negative deflections in the extracellular potential are caused by the depletion of positive ions or by the accumulation of negative ions in the extracellular space and reflect membrane depolarizations of neurons in the ganglion cell layer.

The LFP fundamental frequency was estimated from the power spectral density (PSD) functions computed from long (10 s) calibrated voltage traces. The fundamental frequency was measured as the first peak power in the range $3-100 \mathrm{~Hz}$.

The calculation of LFP propagation velocity is performed in analogy to the velocity calculation of developmental calcium waves (Blankenship et al., 2009). The method requires accurate definition of the LFP boundary. Therefore, each voltage map $\left(1 \mathrm{~mm}^{2}\right)$ recorded by the sensor array at one time point was first spatially filtered (Gaussian filter, $\sigma=10 \mu \mathrm{m}$ ). Timeconsecutive voltage maps were averaged within a window of $2 \mathrm{~ms}$. In each such averaged voltage map, we identified those sensor areas that measured voltages smaller than $-100 \mu \mathrm{V}$ as a "region of interest" (ROI). Contiguous regions were considered to be part of one LFP. The next ROI was calculated after a delay of $10 \mathrm{~ms}$. LFP propagation velocity was computed if consecutive ROIs covered at least one-half of the sensor array ( 1 $\mathrm{mm}^{2}$ ) and lasted $>50 \mathrm{~ms}$. The border of each ROI represents an isotemporal continuous line of the LFP wavefront. The furthest distance traveled during the last time interval was used as an ending point of the propagation path. Points along the propagation path were selected by finding the shortest distance between the point at time $t$ (in milliseconds) and the isotemporal line at time $t-\Delta t$. Velocity was calculated by averaging the distance between consecutive time points $\Delta t$ (values of $\Delta t$ are specified in the legends of Figs. 3, 5, 6). To clarify that the velocity values did not depend on the threshold $(-100 \mu \mathrm{V})$ or the time steps $\Delta t$, we calculated velocities for a subset of waves using a threshold of $-50 \mu \mathrm{V}$ and time steps between 7 and $15 \mathrm{~ms}$, respectively. We obtained qualitative similar results.

Statistical analysis. We could not rigorously test whether the distributions of the various parameters (firing rate; correlation coefficients; LFP velocity; maxima of the power spectral density) follow a normal distribution. To test for statistical significance, we therefore compared median values using the Wilcoxon-Mann-Whitney $U$ test. For the firing rate and correlation coefficient, we present mean values as they are not different from the median.

Pharmacology. To block AMPA/kainate receptors, 6,7-dinitroquinoxaline-2,3-dione disodium salt (DNQX) was used. NMDA receptors were blocked by DL-2-amino-7-phosphonoheptanoic acid (AP-7). Glycinergic synapses were blocked by strychnine, whereas for $\mathrm{GABA}_{\mathrm{A}}$ receptors SR-
95531 hydrobromide (gabazine) [6-imino-3-(4-methoxyphenyl)-1(6H)pyridazinebutanoic acid hydrobromide] was used. Gap junctions were blocked by either meclofenamic acid (MFA) or carbenoxolone (CBX). All antagonists were purchased from Tocris Bioscience. Sodium channels were blocked using tetrodotoxin (TTX) (Sigma-Aldrich).

\section{Results}

We recorded spontaneous electrical activity from isolated adult rd1 retinas using a multi-transistor sensor array (Eversmann et al., 2003; Lambacher et al., 2004). The sensor distance of $7.8 \mu \mathrm{m}$ represents a considerable improvement over existing multielectrode arrays and allows for the precise spatial mapping of electrical activity in the retinal ganglion cell layer. Extracellular signals originating from RGC action potentials were identified using an appropriate filter range $(0.1-3 \mathrm{kHz})$ and taking advantage of the simultaneous recording on many adjacent electrodes. In the low frequency range $(1-60 \mathrm{~Hz})$, we detected extracellular voltage modulations that are caused by the depletion and accumulation of ions in the extracellular space, respectively. Low-frequency changes of the extracellular potential that appear simultaneously on adjacent sensors constitute local field potentials.

\section{The majority of identified retinal ganglion cells in $r d 1$ retinas exhibit rhythmic activity}

Mouse models of retinal degeneration mimic the disease of retinitis pigmentosa, both on the anatomical and physiological level (Chang et al., 2002). Although the gross morphology of the retinal output neurons - the ganglion cells_-remains intact in the rd1 mouse (Strettoi et al., 2002; Margolis et al., 2008; Mazzoni et al., 2008), recent studies report increased spontaneous activity in rd1 ganglion cells (Ye and Goo, 2007; Margolis et al., 2008; Stasheff, 2008) and rhythmic spiking activity in three morphology identified cell types (Margolis et al., 2008). Here, we confirm and extend this finding in adult $r d 1$ retinas (P35-P70) from two different $r d 1$ strains ( $\mathrm{C} 3 \mathrm{H}$ and $\mathrm{FVB} / \mathrm{N}$, respectively).

The spontaneous activity of 20 selected RGCs recorded simultaneously in one retinal portion is shown in Figure $1 a$. The presented RGCs display a rhythmic bursting activity visible in the spike trains autocorrelation function (Fig. 1b). High-passfiltered $(0.1-3 \mathrm{kHz})$ calibrated voltage traces of selected RGCs are shown in supplemental Figure 1 (available at www.jneurosci.org as supplemental material). Some of the rhythmic RGCs do not elicit bursts continuously; however, their autocorrelation functions remain rhythmic. Within the retina presented in Figure $1 a-d$, the spiking of most RGCs (90\%) occurred with the same fundamental frequency $(7.5 \pm 0.3 \mathrm{~Hz}$; mean $\pm \mathrm{SD} ; n=70$ RGCs). The average fundamental frequency calculated for 403 rhythmic RGCs ( $n=7$ retinas) was $9.2 \pm 1.8 \mathrm{~Hz}$ (mean $\pm \mathrm{SD}$ ). This result was obtained from both $r d 1$ mouse strains used in this study. RGCs in older retinas (C3H/HeNCrl; P160-P200; $n=110$ RGCs in three retinas) display rhythmic activity with an average fundamental frequency that is not significantly different from the values calculated for the younger retinas $(8.8 \pm 1.3 \mathrm{~Hz}$; mean \pm $\mathrm{SD} ; p=0.22)$. The percentage of rhythmic RGCs is high across all $r d 1$ retinas (85\% of RGCs in P35-P70) and declines slightly in older retinas (75\% of RGCs in P160-P200). The average firing rate (Table 1) of spontaneous activity in $r d 1$ RGCs (P35-P70) is significantly higher $(p<0.01)$ than in old $r d 1$ RGCs (P160P200), in agreement with previous reports (Margolis et al., 2008; Stasheff, 2008). The absolute value of spontaneous firing rate in rd1 RGCs is in line with Margolis et al. (2008) but higher than the value reported by Stasheff (2008). This may be attributed to slightly different recording conditions such as perfusion medium or perfusion rate. 
a

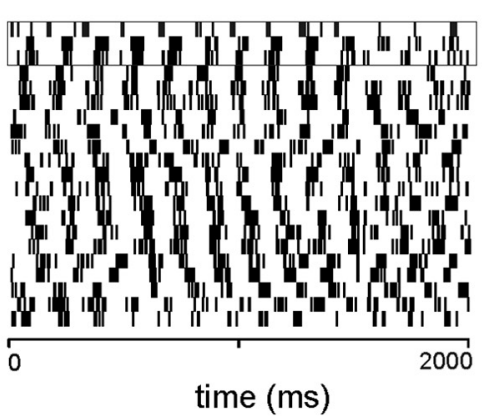

e

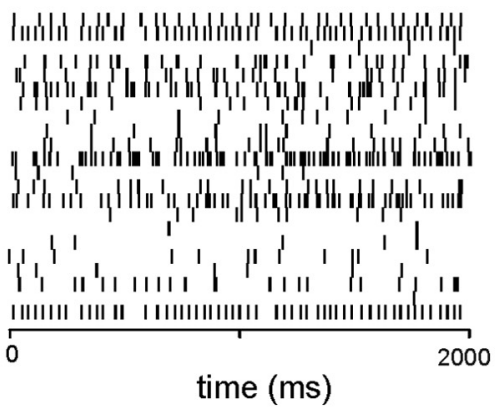

b

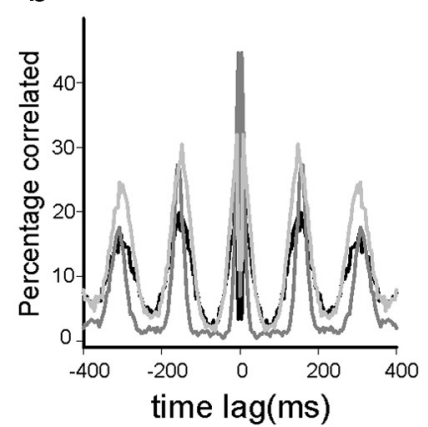

f

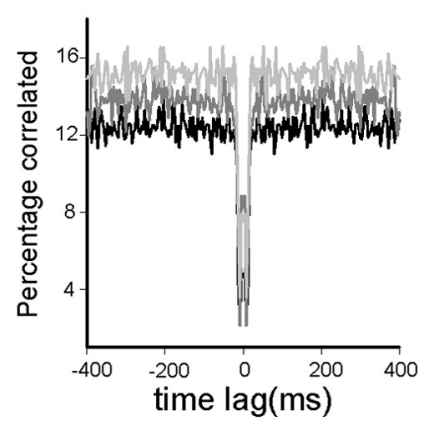

C

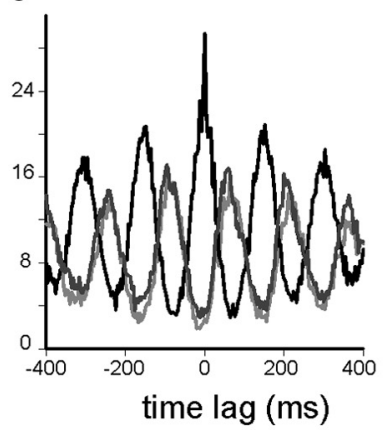

g

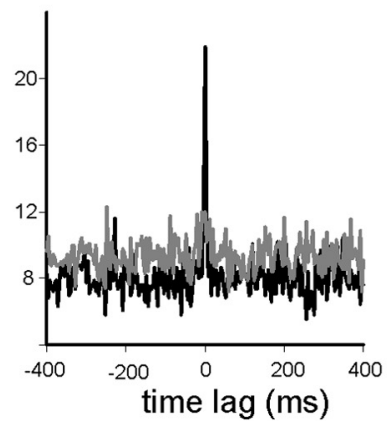

d

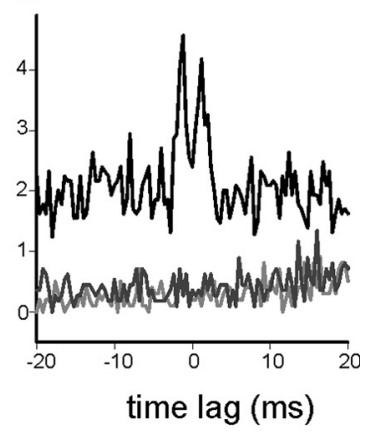

h

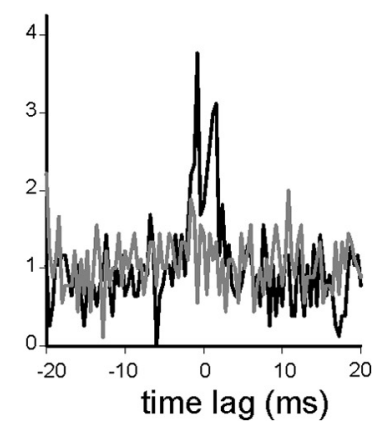

Figure 1. The majority of RGCS in the $r d 1$ mouse retina exhibit rhythmic spiking. $\boldsymbol{a}-\boldsymbol{d}$, Spike train properties of RGCs recorded in a $1 \mathrm{~mm}^{2}$ portion of an rd1 retina. $\boldsymbol{a}$, Spiking activity from 20 selected RGCs. Each tick represent the occurrence of one action potential. The three RGC spike trains in the top row are further evaluated. $\boldsymbol{b}$, Autocorrelation functions of the three selected $r d 1 \mathrm{RGCS}$ reveal rhythmic activity with an average interval of $\sim 150 \mathrm{~ms}$. The peak at zero time lag is omitted. $c$, The spike train CCs between three selected RGCs reveal strong oscillations. For two cell pairs, the activity is phase-shifted. One RGC pair fires in synchrony revealed by the central peak at zero time lag. Bin size, $4 \mathrm{~ms}$. $\boldsymbol{d}$, The same C(s as shown in c, computed at higher resolution (bin size, 0.4 $\mathrm{ms})$. A double peak in one (C at zero time lag indicates electrical coupling between the two RGCs. $\boldsymbol{e}-\boldsymbol{h}, \mathrm{RGC}$ spike train properties in wt retinas. $\boldsymbol{e}$, Raster plot of spontaneous activity. $\boldsymbol{f}$, Autocorrelation function of three selected RGCS. $\boldsymbol{g}$, Spike train cross-correlations between the three selected RGCs shown in $\boldsymbol{f}$. Synchronous activity is detected in one cell pair. Bin size, 4 ms. $\boldsymbol{h}$, The same CCs shown in $\boldsymbol{g}$, computed at higher resolution, reveal a double peak in one C( around origin, similar to the result in the $r d 1$ retina (d). Bin size, $0.4 \mathrm{~ms}$.

Table 1. Properties of retinal ganglion cells and local field potentials in rd1 retinas

\begin{tabular}{lcll}
\hline & $\begin{array}{l}\text { Spontaneous firing } \\
\text { rate }(\mathrm{Hz})\end{array}$ & $\begin{array}{l}\text { Fundamental spiking } \\
\text { frequency }(\mathrm{Hz})\end{array}$ & $\begin{array}{l}\text { LFP velocity } \\
(\mathrm{mm} / \mathrm{s})\end{array}$ \\
\hline Control $w$ t retina & $17 \pm 10$ & - & - \\
Untreated $r$ 1 1 retina & $26 \pm 13$ & $9.2 \pm 1.8$ & 8 \\
TTX-treated $r d 1$ & 0.2 & $5^{a}$ & 6.6 \\
DNQX-treated $r d 1$ & $15.5 \pm 4$ & - & - \\
Strychnine/gabazine-treated $r d 1$ & $22 \pm 10$ & $6.7 \pm 0.8$ & 6 \\
\hline
\end{tabular}

Data of firing rate and fundamental frequency are given as mean \pm SD. Data for the LFP propagation velocity represent the median of the distribution.

${ }^{a}$ Fundamental frequency calculated from the LFP power spectra.

We could not detect any rhythmic activity in spontaneously active RGCs from $w t$ retinas (Fig. $1 e, f$ ) recorded with the same multitransistor array under otherwise identical recording conditions $(n=$ 200 RGCs; $n=7$ retinas; age, P35-P60). This result applied to both $w t$ mouse strains used in this study. We note, in agreement with previous studies (Margolis et al., 2008; Stasheff, 2008), that the spontaneous firing rate of $w t$ RGCs is significantly lower $(p<0.01)$ than the firing rate of $r d 1$ RGCs of the same age (Table 1).

\section{The majority of rhythmic $r d 1$ RGCs display}

phase-shifted activity

In the following, we investigate whether all RGCs in one retinal portion fire in synchrony. The cross-correlograms (CCs) between pairs of $r d 1$ RGC spike trains display multiple peaks (Fig. 1c) separated by $100-150 \mathrm{~ms}$. In most CCs (86\%; $n=1688$ analyzed CCs within one retina), the correlation peak does not occur at zero time lag (Fig. 2) but at any value between -70 and $70 \mathrm{~ms}$.
This variability suggests that either the RGCs in the recorded retinal portion do not receive the same synchronous input or that each RGC exhibits a different spike threshold.

We first analyze CCs that show a central peak at zero time lag. When computed at high resolution, several "peaked" CCs displayed two subpeaks around zero time lag (Fig. 1d). The two maxima were located on average at $-1.4 \pm 0.2$ and $1.3 \pm 0.3 \mathrm{~ms}$, respectively ( $n=40$ pairs in three retinas). These peaks are a strong indicator of reciprocal electrical coupling between RGCs, where the spike in one cell depolarizes the coupled cell above spiking threshold. Comparable double-peaked synchronization patterns have been described in electrically coupled RGCs in healthy mammalian retinas (Mastronarde, 1989; Hu and Bloomfield, 2003). The average distance between electrically coupled rd1 RGCs is $120 \pm 30 \mu \mathrm{m}$ (range, $60-180 \mu \mathrm{m}$ ). This indicates that only nearby RGCs are electrically coupled. The average correlation strength of these pairs is $0.20 \pm 0.05$ (mean $\pm \mathrm{SD}$; bin width, $4 \mathrm{~ms}$ ). The correlation strength does not vary over the distance mentioned above. In CCs calculated from $w t$ RGC spike trains, we also find peaked CCs $(n=15)$ that display two subpeaks around zero time lag (Fig. 1g,h). The CC maxima were located on average at $-1.8 \pm 0.4$ and $1.7 \pm 0.3 \mathrm{~ms}$, respectively, whereas the spacing between cells was $133 \pm 40 \mu \mathrm{m}$ (range, 66-224 $\mu \mathrm{m}$ ). The average correlation strength of these pairs is $0.10 \pm 0.04$ (mean \pm SD; bin width, $4 \mathrm{~ms}$ ). The correlation coefficients in $w t$ RGCs are statistically different $(p<0.001)$ from those measured for $r d 1$ RGCs. We conclude that the subpopulation of electrically coupled RGCs in $r d 1$ and $w t$ retinas have similar properties, except for the stronger electrical coupling strength between $r d 1$ RGCs. 

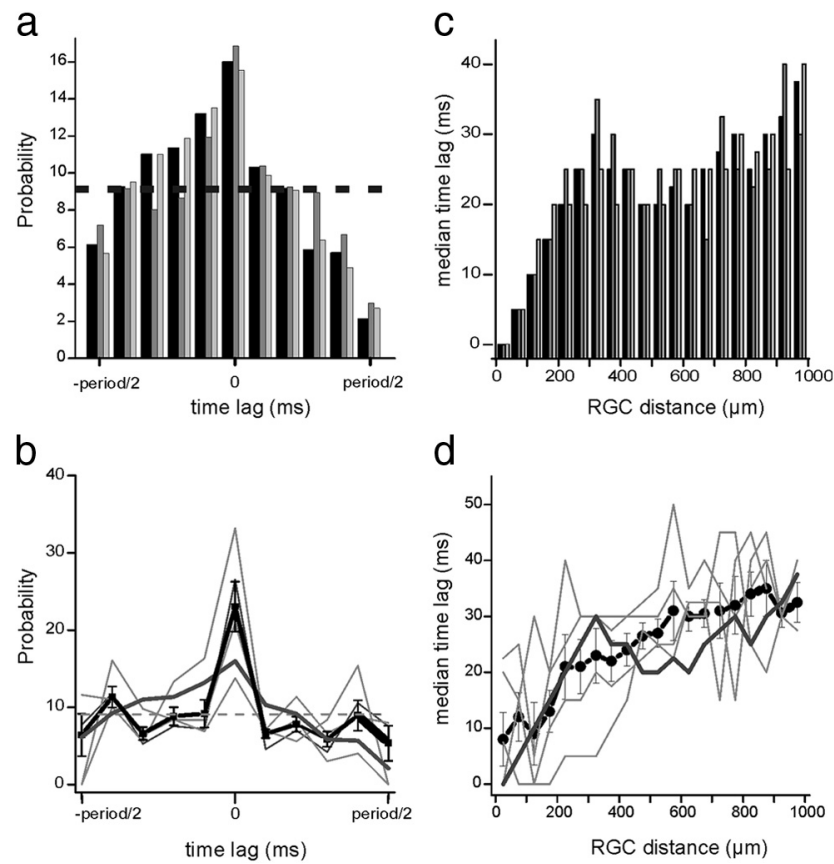

d

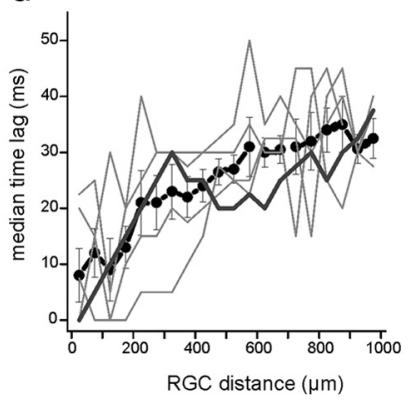

Figure 2. Nearby RGCs in $r d 1$ retinas oscillate with little time lag. $\boldsymbol{a}$, Histogram of the time lags of the central CC peaks of RGC spike trains calculated within one $r d 1$ retina (2 CCs were presented in Fig. 1c). The dashed line marks chance level. The dark gray bars mark the result obtained if the first half of recording is considered; the light gray bars, the results obtained from the second half of recorded data. Bin size, $10 \mathrm{~ms}$. Each CC time lag is normalized to the average rhythm period. $\boldsymbol{b}$, Average of five CC histograms calculated as in $\boldsymbol{a}$. With the exception of zero time lag, there is no preference for any phase shift of the central CC peak. Each gray line represents the result from one retina. The thick gray line represents the data shown in $\boldsymbol{a}$. The black symbols mark the mean values for a given time lag. The dashed line represents uniform distribution. c, Dependence of the peak CC time lag on the distance between RGCs. The analysis was performed on the same dataset shown in $\boldsymbol{a}$. Up to separation distance of $\sim 300 \mu \mathrm{m}$, the median time lag increases with increasing distance. The black bars denote the median values obtained for the whole recording session. The dark gray bars mark the result obtained if the first half of recording is considered; the light gray bars, the results obtained from the second half of recorded data. No significant changes are measured. Bin size, $50 \mu \mathrm{m}$. d, Average of five histograms of median time lags calculated as in c. The same dataset of the five retinas evaluated in $\boldsymbol{b}$ was used. The thick gray line represents the data shown in c. The black symbols mark the median time lag for a given RGC distance. The tendency of short time lags for RGC separations $<500 \mu \mathrm{m}$ is preserved across retinas.

We return to the analysis of the $r d 1$ spike train CCs. As mentioned, the majority of spike train CCs (range, $60-86 \% ; n=5$ retinas) have their most central peak shifted with respect to zero time lag (Figs. 1c,2). We therefore tested whether there was any preferred phase shift between RGC spiking and whether the phase shift depends on the RGC separation. As the fundamental RGC spiking frequency differs among retinas (range, 7-10 Hz), we calculate for each retina an average rhythm. We then normalize each CC time lag to the retina specific rhythm. A time lag of 50 ms between two RGCs spiking at a fundamental frequency of 10 $\mathrm{Hz}$ corresponds to a relative shift of period/2, whereas the same absolute time shift gives a smaller relative shift for RGCs spiking at $7 \mathrm{~Hz}$. First, the probability to measure a shifted CC peak is close to chance level for any value within one period, with the exception of zero time lag (Fig. $2 a, b)(n=5$ retinas). The average probability varies little if either half of the recording session is evaluated (Fig. 2a), although for individual CCs a small shift of the central peak is observed (data not shown). Second, for all RGCs separated by $<500 \mu \mathrm{m}$, the median value of the central CC peaks increases from $8 \mathrm{~ms}$ (RGC separation, $<50 \mu \mathrm{m}$ ) to $25 \mathrm{~ms}$ (RGC separation, $500 \mu \mathrm{m}$ ) (Fig. 2c). For separation distances
$>500 \mu \mathrm{m}$, the peak time lags occur at any value with nearly equal probability (Fig. $2 d$ ).

These findings point toward a local driving force of the rhythmic spiking of $r d 1$ RGCs. In the next paragraph, we therefore investigate whether this driving force is caused by independent, "pacemaker-like" cells, as might be inferred from Figure 2, $a$ and $b$, or whether spatial interactions (suggested by Fig. $2 c, d$ ) shape the oscillatory behavior.

\section{The rhythmic spiking of $r d 1$ RGCs correlates with local field potential minima}

All sensors recording rhythmic $r d 1$ RGC spiking display a modulation of the extracellular voltage in the low-frequency (1-60 $\mathrm{Hz}$ ) range (Fig. 3a). Voltage modulations that occur in phase across nearby sensors reflect spatially confined local field potentials (Fig. 3b). Negative deflections in the extracellular potential are caused by the depletion of positive ions or the accumulation of negative ions in the extracellular space, as reported in other neural tissues (Mitzdorf, 1985). LFPs were never found in $w t$ retinas under the same recording conditions.

LFPs initiated at several locations across the $r d 1$ retina. During the recording time $(1-3 \mathrm{~h})$, the entire portion of the imaged ganglion cell layer was part of a LFP. Continuous (10 s) readout of the sensors was used for PSD analysis indicating a fundamental LFP frequency of $7-10 \mathrm{~Hz}$ (Table 1). LFP and spiking activity was interleaved with periods of silence (supplemental Fig. 1, available at www.jneurosci.org as supplemental material).

To investigate whether LFPs are responsible for rhythmic RGC spiking, we correlated the spike trains of individual RGCs with the timing of LFP minima recorded in the vicinity of the ganglion cells. Different temporal filters for action potential $(0.1-3 \mathrm{kHz})$ and LFP $(0-60 \mathrm{~Hz})$ identification, and a distance of $32 \mu \mathrm{m}$ between the RGC recording sensor and the LFP recording sensor guarantees that RGC spikes do not influence the LFP minima. The cross-correlation of three selected LFP-RGC pairs revealed an oscillatory function peaked around zero lag (Fig. $3 c$ ). The analysis of 188 LFP-RGC pairings in three retinas reflects that most CC peaks occur in the interval around zero lag. The majority $(75 \%)$ of the CC peaks occur at a time lag between -12 and $2 \mathrm{~ms}$ (Fig. 3d).

\section{Propagation of local field potentials}

Local field potentials are dynamic: they expand (Fig. 4) or propagate across the retinal surface (Fig. 3) but never collapse. Occasionally, we observed the initiation of two LFPs within the imaged area. Within one retina, consecutive wave-like LFPs propagated in the same direction (Fig. $3 b$ ). Because the propagation of LFPs resembled activity patterns seen in immature retinas, we adopted methodology used to assay the speed of developmental waves (Blankenship et al., 2009). We consider LFPs averaged over $2 \mathrm{~ms}$ of electrical activity. In each such averaged voltage map, we identified those sensor areas that measured voltages smaller than $-100 \mu \mathrm{V}$ as a ROI. The next ROI was calculated after a fixed delay $\Delta t$ (Fig. 4). The identified ROIs propagated across the retina with a median velocity of $8 \mathrm{~mm} / \mathrm{s}$ ( $n=292$ waves in five retinas; for details of velocity calculation, see Materials and Methods). The propagation of electrical activity could, in principle, be assessed using the RGC spiking. However, because RGCs started a burst (3-10 action potentials) every 100-150 ms, the methodology used in extracellular recordings of developing retinas (Demas et al., 2003), which display much longer interburst intervals, failed here.

The analysis of RGC spiking and LFP propagation suggests that RGC depolarizations occur through a local mechanism that 

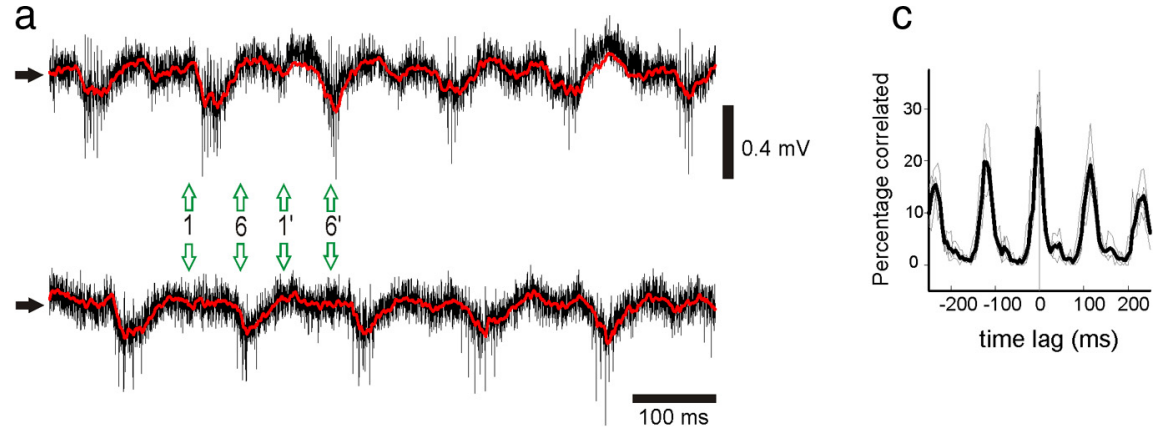

b
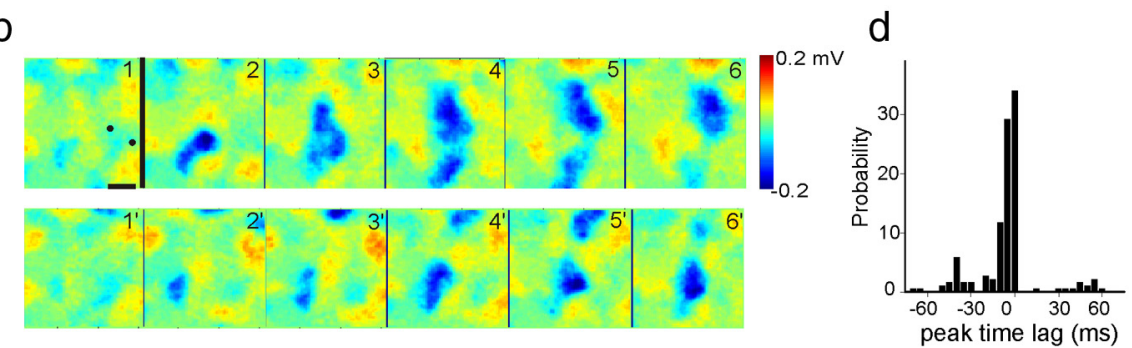

Figure 3. RGC spiking and local field potential minima coincide in $r d 1$ retinas. $a$, Extracellular voltage recordings from two sensor electrodes separated by $\sim 300 \mu \mathrm{m}$. The filter settings of $1 \mathrm{~Hz}$ to $3 \mathrm{kHz}$ reveal single spikes and a slow oscillatory extracellular potential. The sensor locations are marked in subplot b1. Red trace, Low-pass-filtered (1-60 Hz) signals reveal rhythmic LFPs. The horizontal arrow marks zero extracellular potential. The open green arrows mark the start point and end point of a six frame series of extracellular voltage maps, shown in $\boldsymbol{b}$. $\boldsymbol{b}$, Extracellular voltage maps ("electrical images") recorded at a spatial resolution of $8 \times$ $16 \mu \mathrm{m}$. Each image represents the average extracellular voltage over $2 \mathrm{~ms}$. Separation between images is $10 \mathrm{~ms}$. Scale bar, 200 $\mu \mathrm{m}$. c, Cross-correlation of three spike trains with LFP minima revealing central peaks with minimal time lag (gray). The average of the three traces is shown as a thick black line. LFP minima were evaluated for sensors separated by $32 \mu \mathrm{m}$ from the RGC recoding sensor. $\boldsymbol{d}$, Distribution of peak CC time lags for RGCs and nearby recorded LFPs in two retinas. The recordings presented here were from a different $r d 1$ retina as shown in Figure 1.

spreads laterally across the retina. To identify circuitry involved in the generation and propagation of LFPs and concomitant RGC spiking, we applied pharmacological agents that disrupted either RGC spiking and/or LFPs.

\section{Local field potentials persist when voltage-gated sodium channels or inhibitory receptors are blocked}

We first excluded the hypothesis that spontaneous sodium spikes are responsible for the extracellular LFPs by adding the sodium channel blocker TTX $(0.2 \mu \mathrm{M})$ to the perfusion solution. Under these conditions, we measured LFPs but no RGC spikes (Fig. $5 a, b)$. The sodium channel blocker abolished the RGC firing in all but seven RGCs from a total of 138 identified cells in three retinas. The RGC firing rate dropped to $0.3 \mathrm{~Hz}(n=7$ RGCs in three retinas). The LFP amplitude measured as the peak of the voltage power spectrum (Fig. $5 b$ ) did not change after application of TTX. We quantified LFP amplitudes on 20 sensors that recorded from the same retina before and after the application of TTX. The average of the maximum power spectral densities was not different $(p=0.1)$ in the two conditions (Fig. $5 b$, inset). Similarly, no visible changes occur in the overall LFP shape (Fig. $5 c$ ). However, the LFP propagation velocity (Table 1) decreased to $6.6 \pm 2.6$ $\mathrm{mm} / \mathrm{s}$ (mean $\pm \mathrm{SD} ; n=55$ waves; statistical significance, $p<$ 0.05) (Materials and Methods). The LFP fundamental frequency decreased from an average of 9 to $6.2 \mathrm{~Hz}$ calculated on 16 sensors in the three retinas (one trace shown in Fig. 5b). After washout, the rhythmic sodium spikes appeared again in all three retinas tested. This experiment proves that, although sodium channels shape certain LFP properties, the RGC action potentials do not contribute to LFPs. Thus, neurotransmitters acting on nonspecific ion channels may be responsible for the LFP generation.
In a second experiment, we investigated the contribution of inhibitory circuits on the generation of LFPs and rhythmic RGC activity. In the $r d 1$ retina, the two inhibitory cell classes are preserved to a very different degree-horizontal cells retract axonal and dendritic processes, whereas amacrine cells appear morphologically well preserved (Strettoi and Pignatelli, 2000; Park et al., 2001; Strettoi et al., 2002). Amacrine cells inhibit synaptically connected bipolar cells or RGCs by the release of either GABA or glycine (Wässle, 2004). Horizontal cells modulate photoreceptor output through feedback mechanisms that are still debated (Wässle, 2004). The application of the combination of glycinergic receptor blocker strychnine $(20 \mu \mathrm{M})$ and $\mathrm{GABA}_{\mathrm{A}}$ receptor blocker gabazine $(40 \mu \mathrm{M})$ had the following effects on RGC spiking and LFPs. The average firing rate (Table 1$)$ of RGCs did not change significantly ( $p=$ 0.09 ) in the presence of inhibitory receptor blockers. Blocking inhibitory receptors led to the synchronization of more RGCs (Fig. 6b,c). The central peak in the spike CCs occurred with a time lag of $<5$ $\mathrm{ms}$ in $43 \%$ of the CCs (average over RGCs in five retinas) compared with $23 \%$ in untreated retinas (Fig. 2b). Within each retina, the probability of synchronous RGC activity increased on average twofold. RGC spike trains and LFP minima correlated with low time lag in the presence of inhibitory receptor blockers, as was observed for the unperturbed state (compare Figs. $6 d, e, 3 c, d$ ). The spatial extent and extracellular amplitude of LFP increased after disinhibition of the retina (Fig. $6 f)$. However, we never measured simultaneous low-frequency oscillations covering the whole sensor array $\left(1 \mathrm{~mm}^{2}\right)$, both in disinhibited and also in unperturbed $r d 1$ retinas. The fundamental RGC spiking frequency (Table 1), the LFP frequency, and the LFP conduction velocities decreased compared with the unperturbed state significantly $(6 \pm 2 \mathrm{~mm} / \mathrm{s}$; mean $\pm \mathrm{SD} ; n=110$ waves; $p<$ $0.05)$. The application of each inhibitory blocker alone revealed that strychnine ( $2 \mu \mathrm{M} ; n=2$ retinas) had a stronger effect on the LFPs than gabazine ( $40 \mu \mathrm{m} ; n=2$ retinas) (supplemental Fig. 2, available at www.jneurosci.org as supplemental material). The average LFP amplitude-measured as the peak of the voltage power spectrumincreased significantly $(p<0.001)$ when glycinergic receptors were blocked. The LFP amplitude did not increase significantly when only $\mathrm{GABA}_{\mathrm{A}}$ receptors were blocked $(p=0.07)$. Strychnine reduced the fundamental LFP frequency to a larger extent than gabazine (supplemental Fig. 2, available at www.jneurosci.org as supplemental material).

These results indicate that, although inhibitory neurons shape the physical LFP properties, they do not contribute to their generation. The results suggest that, in the unperturbed $r d 1$ retinas, glycinergic and GABAergic inhibition prevents the recruitment of additional excitatory neurons that depolarize the RGCs.

\section{Local field potentials require glutamatergic input to RGCs}

The excitatory neurotransmitter glutamate is used at several types of synapses in the retina: in the outer retina at the photoreceptor- 


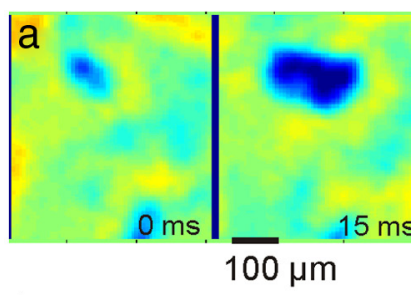

b

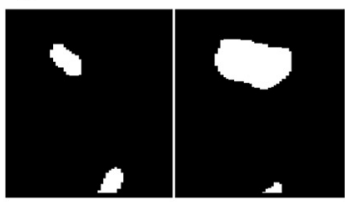

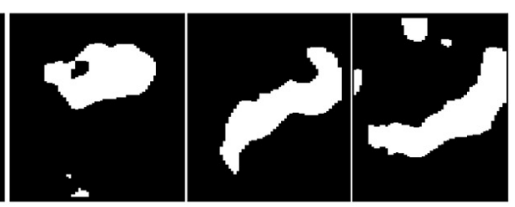

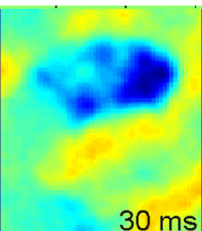

$30 \mathrm{~ms}$

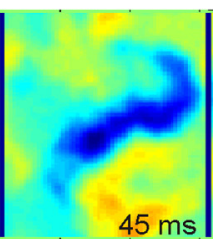

$45 \mathrm{~ms}$

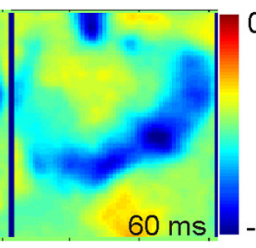

$0.2 \mathrm{mV}$

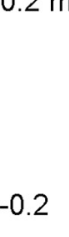

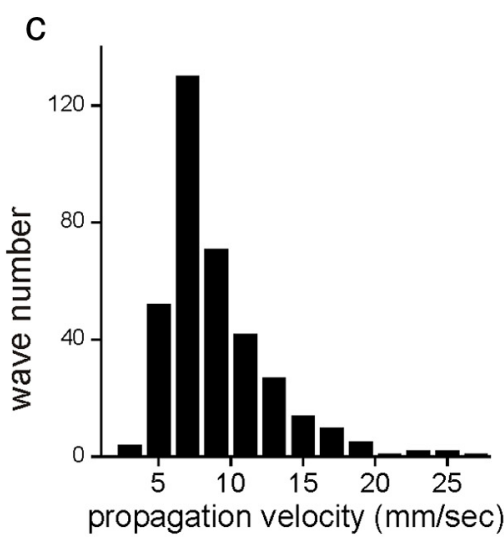

Figure 4. Local field potentials propagate across the retina. $a$, Extracellular voltage maps recorded at time intervals of $15 \mathrm{~ms}$. Each image represents the average activity over $2 \mathrm{~ms}$. The activity was recorded in the same retina shown in Figure $3 b$, but recorded at a later time. $\boldsymbol{b}$, For the estimation of the wave-like LFP propagation velocities (see Materials and Methods), we identified region of interest with voltages more negative than $-100 \mu \mathrm{V}$. Each binary image represents the region of interest calculated for each voltage map shown in $\boldsymbol{a}$. Right frame, Spatial extension of LFP propagation. Each grayscale represents the region of interest at one of the five time points. Two arbitrary points are shown that were selected to trace back the propagation paths (red). $c$, Histogram of LFP propagation velocities. A total of 292 LFPs within five retinas were evaluated. Bin size, $2.5 \mathrm{~mm} / \mathrm{s}$. Median value, $8 \mathrm{~mm} / \mathrm{s}$.

bipolar cell synapse and in the inner retina at the synapses between bipolar cells, amacrine cells, and ganglion cells (Thoreson and Witkovsky, 1999; Wässle, 2004). In the retina, glutamate is sensed by ionotrophic (AMPA/kainate and NMDA) receptors and by metabotropic receptors. We therefore checked whether glutamate induced transmembrane currents are responsible for rhythmic RGC spiking. The application of a ionotrophic glutamate receptor (iGluR) blocker mixture $(100 \mu \mathrm{M}$ DNQX/20 $\mu \mathrm{M}$ AP-7) led to the disappearance of LFPs (compare Fig. $7 a, c)(n=4$ retinas). This change was accompanied by a significant decrease of the RGC firing rate (Table 1) and the disappearance of rhythmic spike trains. After the washout of the receptor blockers, the majority of RGCs (80\% in the considered RGC population) recovered their rhythmic activity. In control $w t$ retinas, the application of the iGlu receptor blockers abolished the RGC spontaneous activity.

Why do $r d 1$ RGCs continue to spike after inhibition of their major presynaptic input? The retinas treated with the iGluR blocker mixture revealed electrically coupled RGCs that are not seen in the unperturbed $r d 1$ retina. The strong presynaptic input common to neighboring RGCs may obscure the electrical coupling in $r d 1$ retinas (Fig. $7 b$ ). Inhibition of the glutamatergic input reveals a sharp peak in the CC at zero time lag (Fig. $7 d$ ), whereas in the untreated retina the same CC displayed a broad peak (Fig. $7 b$ ). When computed at high resolution, the peaked CC displayed two subpeaks around zero lag similar to those seen in unperturbed $r d 1$ and $w t$ retinas (Fig. $1 d, h)$. The maxima for this pair and those of 39 others in three retinas were located on average at $-1.5 \pm 0.3$ and $1.4 \pm 0.3 \mathrm{~ms}$, respectively. The average distance between electrically coupled $r d 1$ RGCs in the presence of iGluR blockers is $128 \pm 38 \mu \mathrm{m}$. The average strength of $0.22 \pm 0.06$ (bin size, $4 \mathrm{~ms}$ ) is similar to the

C the array. b
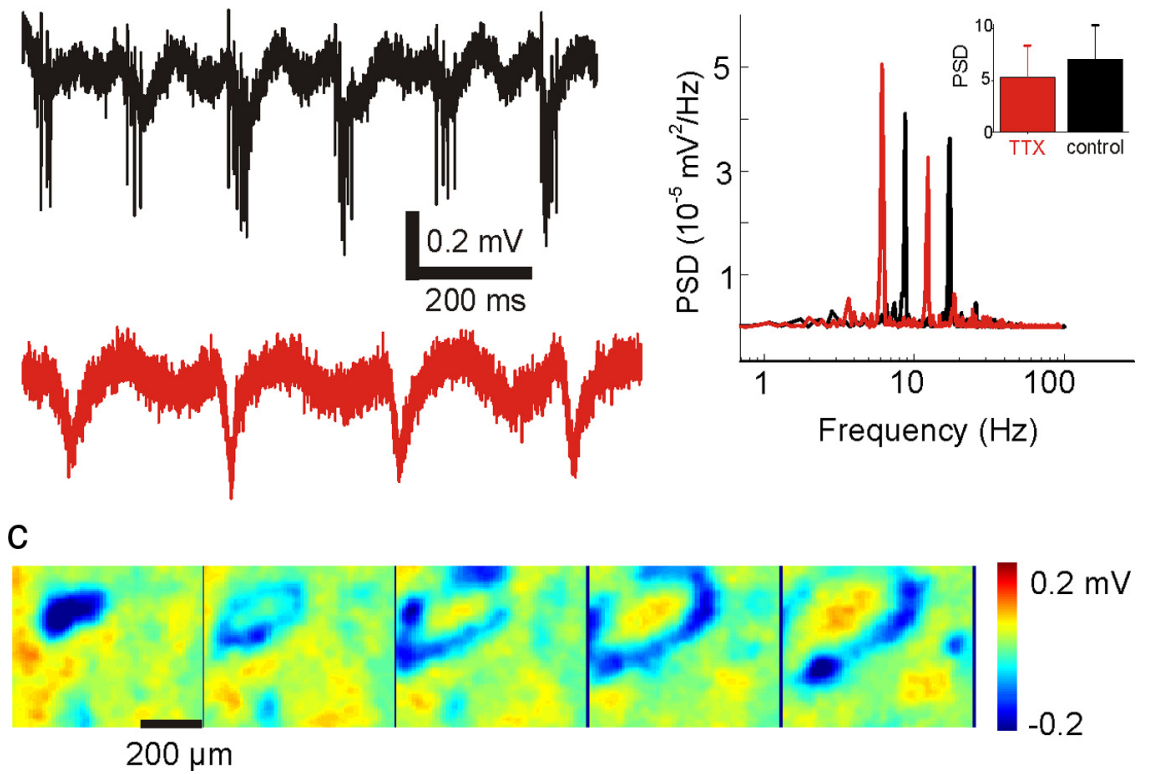

$0.2 \mathrm{mV}$

Figure 5. Application of the sodium channel blocker TTX abolishes RGC spikes but does not inhibit the initiation and propagation of LFPs. $\boldsymbol{a}$, Extracellular voltage recording on one sensor, showing the RGC spikes and LFPs under control conditions (top trace) and after the application of $0.2 \mu \mathrm{m}$ TTX (red trace; bottom). $\boldsymbol{b}$, Power spectral density of the traces shown in $\boldsymbol{a}$ demonstrate that TTX reduces the fundamental LFP frequency. The amplitude of the LFPs, reflected in the PSD, does not change significantly after the application of TTX. Inset, Average peak power measured on 30 sensors before (black) and after (red) the application of TTX. c, Representative electrical images of TTX treated $r d 1$ retina indicating the LFP expansion. Each frame represents the electrical activity averaged over $2 \mathrm{~ms}$. The time interval between frames is $15 \mathrm{~ms}$. The traces shown in $\boldsymbol{a}$ were selected from a sensor in the center of

strength without iGluR blockers $(p=0.26)$. We conclude that rd1 RGCs exhibit nonrhythmic spiking in the presence of iGluR blockers that may be attributed to stronger electrical coupling between nearby RGCs (and possibly retinal interneurons) compared with wt RGCs (coupling strength, 0.01). We infer here the increased coupling strength from higher cross-correlation strength.

The application of AMPA/kainate receptor blockers alone (DNQX) had qualitatively similar results as those reported above. In contrast, the application of the NMDA receptor blocker AP-7 $(100 \mu \mathrm{M})$ alone did not lead to any significant changes in either 
a

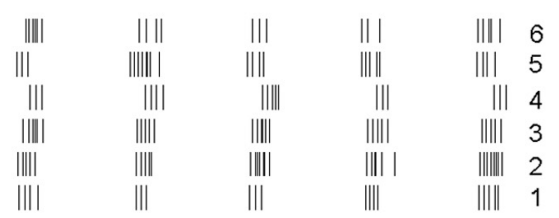

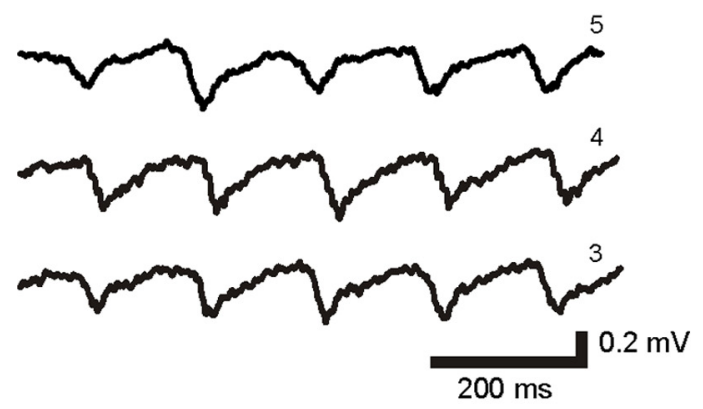

b

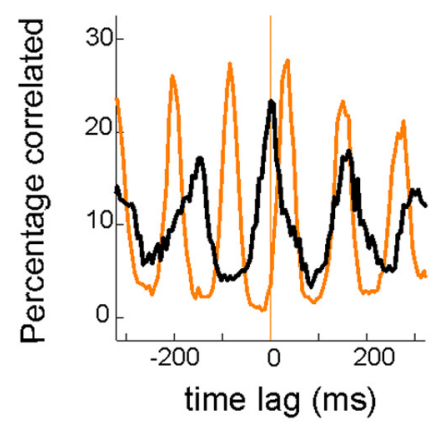

C

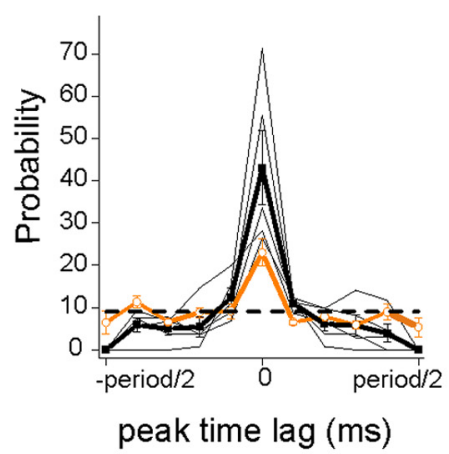

d

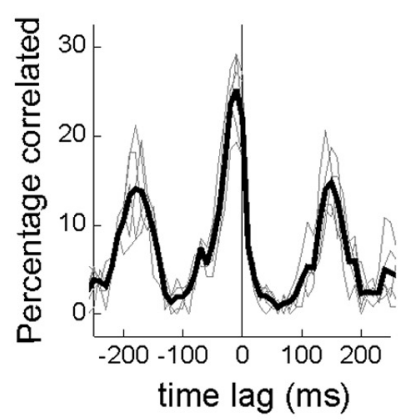

e

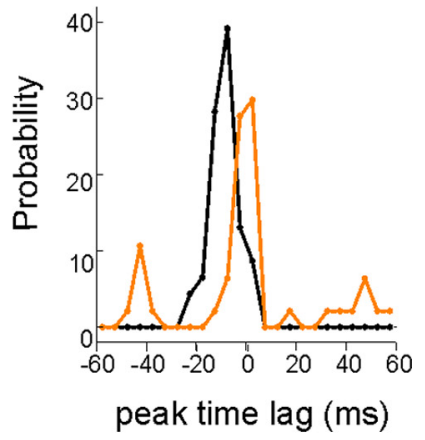

f 8.2 16.4 24.6 32.8

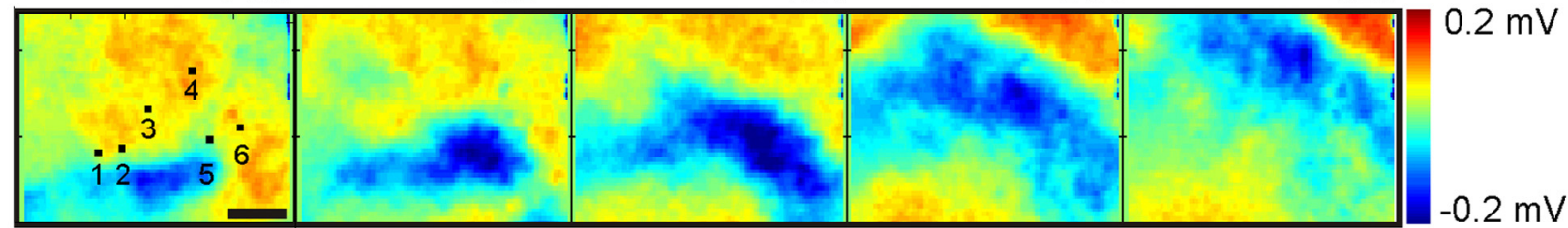

Figure 6. Inhibition of glycinergic and GABAergic receptors increases the LFPs and the synchronization among $r d 1$ RGCs. $\boldsymbol{a}$, Top traces, Spike trains from six RGCs recorded after the application of inhibitory receptor blockers. Each tick marks the occurrence of one spike. The sensor locations are marked in subplot $f$. Bottom traces, LFPs measured near the spike recording sensors (bandpass of $1-60 \mathrm{~Hz}$ ) at three positions (indicated by numbers) on the array. $\boldsymbol{b}$, An example spike train cross-correlogram (CC) before (orange) and after the application of inhibitory receptor blockers (black trace). The CC peak shifts to zero lag when inhibitory receptors are blocked. The CCs were calculated for the spike trains of cells 3 and 4. c, Average probability distribution of minimum time lag of the central CC peak under control conditions (orange) (Fig. 2b), and with inhibitory receptors blocked (black). The distributions are calculated for the same five retinas (gray) that were evaluated in the untreated retinas (Fig. 2b). The uniform distribution is shown as a dashed line. $\boldsymbol{d}$, CC calculated between LFP minima and RGC spike trains for the three LFPs and RGCs shown in $\boldsymbol{a}$. $\boldsymbol{e}$, Distribution of peak time lags for CCs calculated between RGC spike trains and nearby LFPs before (orange) and after (black) the application of glycinergic and GABAergic receptor blockers. The probability distribution was calculated for RGC-LFP pairs in one retina. $f$, Propagation of the LFPs in the presence of inhibitory receptor blockers. Each frame shows the electrical image averaged over $2 \mathrm{~ms}$ (see Materials and Methods). Scale bar, $200 \mu \mathrm{m}$.

RGC spiking or LFP properties ( $n=2$ retinas), indicating that AMPA/kainate receptors are sufficient to mediate the oscillatory behavior. Metabotropic glutamate receptors of type 6 (mGluR6) are expressed in the $w t$ retina at the sign-inverting synapse between photoreceptors and ON bipolar cells (Wässle, 2004). Immunohistochemical evidence suggest a reduced expression of this receptor in $r d 1$ retinas (Chua et al., 2009), whereas a recent functional study using resensitized cones in $r d 1$ retinas proves that the ON pathway is still functional (Busskamp et al., 2010). The mGluR6 receptor antagonist AP-4 $(50-100 \mu \mathrm{M})$ had no effect on either the spontaneous RGC activity ( $n=2$ retinas) or on LFPs (data not shown). In summary, this set of experiments indicates that AMPA/kainate receptors on the RGCs elicit membrane currents that are reflected in our recordings as LFPs. In the following section, we search for a mechanism mediating the LFP propagation.

\section{Gap junctional coupling is required for the propagation of local field potentials}

The median LFP propagation speed of $\sim 8 \mathrm{~mm} / \mathrm{s}$ is higher than the activity propagation measured for developmental retinal waves. Given that the fastest (stage I) developmental retinal waves are inhibited by gap junction blockers (Syed et al., 2004), we tested whether LFP propagation measured here also relies on gap junction coupling.

We investigated the effect of two gap junction blockers, CBX and MFA, on the RGC spiking activity and the occurrence of LFPs. Application of CBX ( $n=3 r d 1$ retinas) at a concentration of $100 \mu \mathrm{M}$ abolished the LFPs and the rhythmic RGC spiking in $r d 1$ retinas (Fig. $8 a$ ). In the presence of CBX, $50 \%$ of the ganglion cells ( 82 of 162) maintained an average firing rate of $7 \mathrm{~Hz}$; however, the spiking was no longer rhythmic. Washout of CBX (20-30 min) restored the LFPs and the rhythmic RGC spiking activity (Fig. $8 a$, right panel). However, not all RGCs recorded before CBX application were identified after the washout. We therefore repeated experiments using the new gap junction blocker MFA (100 $\mu \mathrm{M})$ (Veruki and Hartveit, 2009). The application of MFA ( $n=3 r d 1$ retinas) completely abolished the LFPs and the RGC spiking in $r d 1$ retinas (Fig. $8 b$ ). Washout of MFA ( $\sim 15 \mathrm{~min})$ restored the LFPs and the rhythmic RGC spiking activity (Fig. 8b). All RGCs recorded before the application of MFA were again identified after the washout of MFA. The percentages of rhythmic RGCs before and after the washout of the 
two gap junction blockers are summarized in Figure $8 c$. To check whether RGCs are capable of spiking in the presence of MFA, we added glutamate $(300 \mu \mathrm{M} ; n=2$ $r d 1$ retinas) to the perfusion solution. After the application of glutamate, a large number of RGCs (60 of 90) started to elicit action potentials at an average firing rate of $5 \mathrm{~Hz}$. However, bath-applied glutamate did not restore LFPs and did not elicit rhythmic RGC spiking in the presence of MFA (Fig. 8d). The spike train autocorrelograms and pairwise CCs did not display any peaks (data not shown), indicating that RGCs spike independent. The application of CBX or MFA (both at $100 \mu \mathrm{M}$ ) to $w t$ retinas did not change the spontaneous RGC firing rate. We conclude that glutamate release in the presence of functional electrical synapses is required for the aberrant LFPs and concomitant rhythmic RGC spiking.

\section{Discussion}

The major finding of our study is that, in rod-degenerated, blind mouse retinas, the majority of RGCs exhibit rhythmic spiking that is driven by spatially extended wave-like presynaptic input. The occurrence and propagation of synchronous RGC membrane depolarizations, reflected as LFPs, relies on electrical coupling between retinal neurons.

\section{Phenomenology of the rhythmic RGC activity and the LFPs} Rhythmic activity was recorded in the majority of RGCs in the photoreceptor degenerated retinas investigated. Our current spike sorting and cell assignment algorithm allows for the identification of $\sim 100 \mathrm{RGCs} / \mathrm{mm}^{2}$. The $w t$ retina comprises $\sim 2-3000$ RGCs $/ \mathrm{mm}^{2}$ (Dräger and Olsen, 1981) that can be classified in $\sim 20$ RGC subtypes (Völgyi et al., 2009). This results in $\sim 100-$ 150 RGCs of each type per square millimeter. In adult $r d$ retinas, the RGC number may remain constant (Mazzoni et al., 2008) or may decrease by $\sim 20 \%$ (Grafstein et al., 1972 ) to $\sim 80-100$ $\mathrm{RGCs} / \mathrm{mm}^{2}$ per subtype. It is therefore not possible to infer from our extracellular recordings whether all RGCs subtypes display rhythmic spiking behavior. However, it is unlikely that only one cell type is recorded here, as multisensor arrays detect activity from ON and OFF RGCs in mouse or rabbit retinas with equal probability (Zeck and Masland, 2007; Stasheff, 2008). Rhythmic RGC activity has been found in three morphologically identified rd1 RGCs (Margolis et al., 2008) and at much longer timescales in rod-degenerated rat RGCs (Sauvé et al., 2001; Marc et al., 2003; Sekirnjak et al., 2009; Kolomiets et al., 2010). It is therefore tempting to speculate that photoreceptor degeneration induces pathologic rhythmic activity in the human disease of retinitis pigmentosa.

Photoreceptor-degenerated retinas retain functional synaptic connections with higher visual centers in mice (Bi et al., 2006; Lagali et al., 2008; Lin et al., 2008; Thyagarajan et al., 2010) and humans (Chen et al., 2006; Zrenner et al., 2010). An early study in anesthetized $r d 1$ mice reports rhythmic activity in neurons of the superior colliculus (Dräger and Hubel, 1978) that are postsynaptic to RGCs. Correlated RGC activity in restricted retinal areas driven by ex-
C
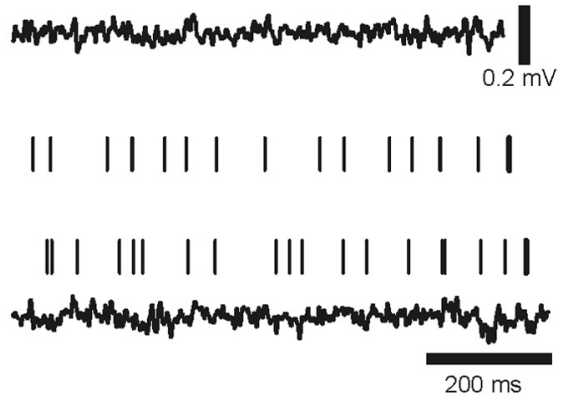

d
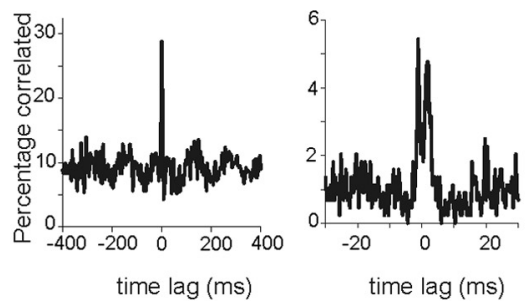

Figure 7. Inhibition of ionotrophic glutamate receptors abolishes LFPs and the rhythmic RGC spiking. $\boldsymbol{a}$, Recording of two . The RGCs are part of the recording shown in Figure $1 a-d . \boldsymbol{b}, \mathrm{CCs}$ of the two $\mathrm{rd1}$ RGC spike trains (shown in $\boldsymbol{a}$ ) reveal a 作

tended LFPs (Figs. 3b, 4, 5) may thus give rise to photopsias reported by RP patients. A high percentage of RP patients describe the sensation of phosphenes or some forms of flashes (Heckenlively et al., 1988; Bittner et al., 2009). We hypothesize the occurrence of phosphenes from the high percentage of synchronous RGC spikes (Fig. 2) that may lead to elevated activity in higher visual areas (Dräger and Hubel, 1978).

Maturation of neuronal circuits is characterized by spontaneous synchronized activity that propagates across extended neural circuits (Wong, 1999; Blankenship et al., 2009). A characteristic feature of developing retinas is concerted RGC activity, reflected as calcium waves or RGC population bursts (Wong, 1999; Blankenship et al., 2009). In developmental waves and in $r d 1$ retinas (Fig. 3), consecutive population bursts are initiated in close proximity. However, in developing retinas, the interburst intervals (seconds to minutes) are much longer compared with the degenerated $r d 1$ retinas $(\sim 100-150 \mathrm{~ms})$. We therefore compare the degenerated retinal circuit with other adult neural circuits exhibiting pathological activity. Perturbations of mature neuronal circuits (i.e., by reducing inhibition) results in aberrant oscillations, hypersynchrony, and eventually the emergence of epileptiform activity (Traub et al., 1993; Pinto et al., 2005). In the $r d 1$ retinas, aberrant activity was detected without perturbing the circuit. However, reducing glutamatergic input abolished LFPs and rhythmic spiking (Fig. 7), suggesting that inhibitory retinal circuits may not be strong enough to compensate the excitatory driving force. Additional disinhibition of the $r d 1$ retinas increased the synchronous discharge of RGCs (Fig. 6; supplemental Fig. 2, available at www.jneurosci.org as supplemental material). Disinhibited neural circuits in the motor cortex generate $\sim 10$ 
a

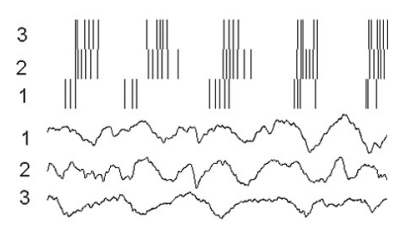

b

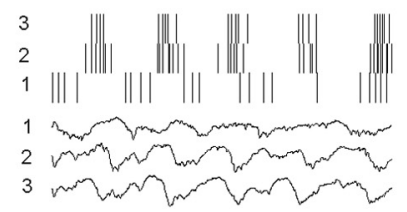

C

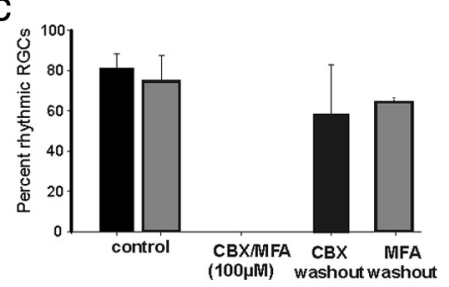

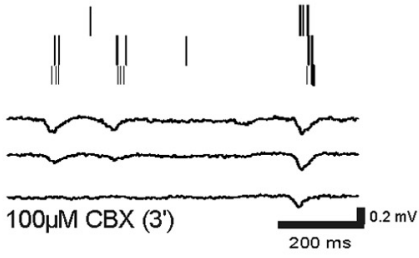
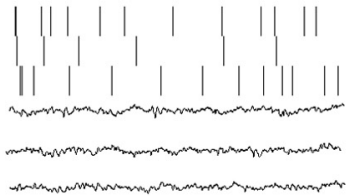

$100 \mu \mathrm{M}$ CBX(6)
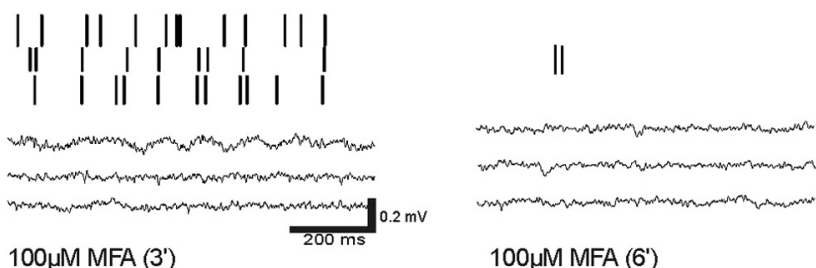

$100 \mu \mathrm{M}$ MFA (6')
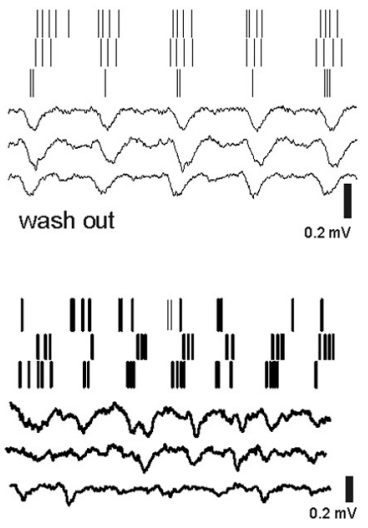

wash out

d

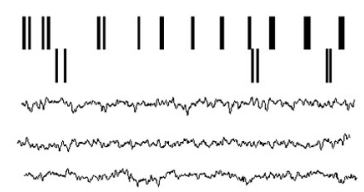

$100 \mu \mathrm{M}$ MFA $+300 \mu \mathrm{M}$ Glutamate

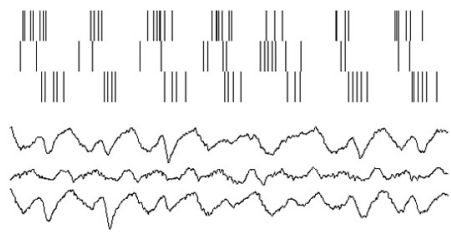

wash out

Figure 8. Application of gap junction blockers abolishes LFPs and the rhythmic RGC spiking. $\boldsymbol{a}$, Effect of the gap junction blocker CBX on RGC spiking and LFPs. Left panel, Raster plot of three rhythmic $r d 1$ RGCs and the LFPs measured $32 \mu \mathrm{m}$ from the soma recordings. The numbers on the left indicate the correspondence between RGCs and LFPs. Middle panels, The application of $100 \mu \mathrm{M}$ CBX abolished rhythmic spiking and reduced the firing rate in the RGCs. The panels represent the recording $3 \mathrm{~min}$ (left) and $6 \mathrm{~min}$ (right) after the application of the CBX. Rhythmic LFPs disappeared gradually during the wash-in of the drug. Right panel, Spike raster plot from the rd1 RGC spikes shown in the left panel measured 20 min after washout of CBX. Rhythmic RGC spiking and LFPs reappear. $\boldsymbol{b}$, The gap junction blocker MFA has similar effects as CBX. Left panel, Control recording of three rd1 RGCs and nearby LFPs. Middle panels, The application of $100 \mu \mathrm{m}$ MFA abolished rhythmic spiking and reduced the firing rate in the RGCs. The panels represent recordings 3 and 6 min after the application of MFA. Rhythmic LFPs disappeared gradually during the wash-in of the drug. Right panel, Rhythmic RGC spiking and LFPs reappear after the washout of the drug. c, In the presence of either CBX or MFA (gray bar), the percentage of rhythmic cells dropped from $\sim 80 \%$ to zero but recovered after washout of the drug. After washout of CBX ( $n=3$ retinas), $60 \%$ of RGCs were rhythmic. After washout of MFA ( $n=4$ retinas), $64 \%$ of the RGCs were rhythmic. $d$, Left panel, Application of glutamate $(300 \mu \mathrm{M})$ in the presence of MFA $(100 \mu \mathrm{M})$ recovered nonrhythmic spiking in $r d 1$ RGCs but not the LFPs. The experiment was performed on the same retina shown in $\boldsymbol{b}$. Right panel, Raster plot of the same three rhythmic $r d 1$ RGCs shown in the left panels and nearby LFPs measured $\sim 30$ min after washout of MFA and glutamate.

$\mathrm{Hz}$ afterdischarge oscillations after each ictal spike (CastroAlamancos et al., 2007). Afterdischarge oscillations in the neocortex (Flint and Connors, 1996) or in disinhibited hippocampal slices (Traub et al., 1993) occur at a similar frequency. Synchronous discharges in these examples rely on the activation of ionotrophic glutamate receptors, and are reflected by strong local field potentials, similar to the results obtained here (Figs. 3, 7).

In $r d 1$ retinas, LFPs expanded and propagated across the retinal ganglion cell layer, depolarizing the RGCs in the corresponding areas. The propagation velocity $(8 \mathrm{~mm} / \mathrm{s})$ is by an order of magnitude higher than the velocities measured across developmental retinal waves in different species $(0.1-1.5 \mathrm{~mm} / \mathrm{s}$ ) (Wong, 1999). The fastest developmental waves (stage I) were abolished by gap junction blockers (Syed et al., 2004) as were the wave-like LFPs in this study. Slower waves (stage III) are suggested to rely on glutamate spillover (Blankenship et al., 2009). This mechanism appears unlikely here. The application of AMPA/kainate antagonists did not block stage III waves, indicating that extrasynaptic glutamate receptors are involved. In the $r d 1$ retinas, LFPs are abolished by AMPA/kainate antagonists.

A rigorous comparison of $r d 1$ LFP propagation speed with various epilepsy models cannot be performed here. However, we mention that the propagation velocity in an epileptiform model that relies on nonsynaptic coupling (Sinha and Saggau, 2001) is in the same range $(10 \mathrm{~mm} / \mathrm{s})$ as the velocity measured here. This brief comparison suggests that aberrant activity in degenerated $r d 1$ retina shares characteristic features with certain forms of epileptiform ac- tivity. However, we note differences between the two disorders. In degenerated $r d 1$ retinas, NMDA receptors and $\mathrm{GABA}_{\mathrm{A}}$ receptors (supplemental Fig. 2, available at www.jneurosci.org as supplemental material) seem to play a minor role, in contrast to many forms of epilepsy (Flint and Connors, 1996; Castro-Alamancos et al., 2007).

\section{Mechanisms underlying the rhythmic RGC activity and the propagating local field potentials}

RGC rhythmic activity could originate within the cells themselves or could be driven by presynaptic input. A previous patch-clamp study established that three identified RGC subtypes are driven by rhythmic presynaptic input (Margolis et al., 2008). Our data are consistent with this result. Rhythmic spiking ceases after the application of iGlu receptors antagonists that suppress excitatory presynaptic input to RGCs (Fig. 7). We therefore infer that RGCs do not act as pacemakers in the $r d 1$ retina. In the adult $r d 1$ retina, glutamate is released by bipolar cells, as the majority of photoreceptors are lost. Recent evidence suggests that bipolar cells may generate a $10 \mathrm{~Hz}$ rhythm in $r d 1$ mouse bipolar cells (Borowska et al., 2010). Indirect evidence from other species [tiger salamander (Gao et al., 2009); goldfish (Protti et al., 2000)] support this hypothesis. Other pacemaker-like interneurons have been reported in the $w t$ retina, such as the starburst amacrine cells (Petit-Jacques et al., 2005) or dopaminergic amacrine cells (Feigenspan et al., 1998). The rhythmic activity of these cells, however, is abolished by TTX, whereas the rhythmic rd1 LFPs persist under TTX application. Our results do not exclude that inhibitory interneurons act as rhythm 
generators (Vaithianathan and Sagdullaev, 2010; Margolis and Detwiler, 2011). However, the pharmacological experiments (Figs. 6, 7; supplemental Fig. 2, available at www.jneurosci.org as supplemental material) demonstrate that $r d 1$ RGCs receive an excitatory rhythmic driving force that is absent in $w t$ retinas. This driving force is modulated but does not rely on inhibitory neurotransmitters.

How does the rhythmic activity propagate across the retina? We discussed that glutamate spillover is unlikely to mediate the activity propagation in $r d 1$ retinas. Our results suggest that gap junctions are involved (Fig. 8). Electrical coupling is abundant in the retina (for review, see Söhl et al., 2005; Bloomfield and Völgyi, 2009) and has been reported between all major neuronal classes. Our recordings demonstrate that the coupling strength between $r d 1$ RGCs is twice as large as that in $w t$ retinas (Fig. 1). Strong electrical RGC coupling persists when iGlu receptors and thus LFPs are blocked (Fig. 7). However, the propagation of membrane depolarizations through strong electrical RGC synapses appears unlikely. The majority of rhythmic RGCs are out of phase (Fig. 2) and do not display electrical coupling (Fig. 1c). Photoreceptors mostly disappeared in the adult $r d 1$ retina (Lin et al., 2009). Horizontal cells are electrically coupled in $w t$ retinas (Hombach et al., 2004; Bloomfield and Völgyi, 2009), but their coverage diminishes during degeneration (Strettoi and Pignatelli, 2000). Among the amacrine cells, one of the most abundant cell types is the glycinergic AII cell (Wässle, 2004). Strychnine strongly modulates the LFPs in $r d 1$ retinas (supplemental Fig. 2, available at www.jneurosci.org as supplemental material), suggesting the contribution of glycinergic cells. AII cells are electrically coupled to ON bipolar cells (Strettoi et al., 1992; Feigenspan et al., 2001) and to neighboring AIIs (Veruki and Hartveit, 2002). Signal transmission between electrically coupled neurons and specifically between AII amacrine cells exhibit low-pass filter characteristics (Veruki and Hartveit, 2002). Lower coupling strength between AII cells results in higher phase shift between paired voltage traces (Veruki et al., 2008). The almost random distribution of CC phase lags between rhythmic RGCs (Fig. 2) may be attributed to variable gap junction coupling between AII cells or between AII and bipolar cells. Indirect evidence reporting the accumulation of glycine in bipolar cells of rod-degenerated rat retinas suggests increased gap junction permeability between bipolar and AII amacrine cells (Fletcher, 2000). Furthermore, TTX inhibits voltage-gated sodium channels in AII cells and slows down the output of the AII network (Tian et al., 2010). This could explain the decreased LFP propagation velocity in TTXtreated $r d 1$ retinas (Fig. 5, Table 1). These arguments suggest the transmission of excitation from rhythmic bipolar cells through a network of electrically coupled amacrine cells. The proposed scenario involving the AII network remains hypothetical; other types of electrically coupled and TTX-sensitive interneurons could mediate the LFP propagation.

An additional experiment is needed to elucidate the transition from normal physiological network dynamics in young, seeing $r d 1$ retinas to the aberrant pathological activity reported here. If the $r d 1$ pathology transfers to the human disease of retinitis pigmentosa, then therapies may aim to reduce aberrant RGC activity by interfering with the increased electrical coupling. The activation of (light-sensitive) $r d 1$ RGCs alone is not sufficient to restore visual percept (Thyagarajan et al., 2010).

\section{References}

Bi A, Cui J, Ma YP, Olshevskaya E, Pu M, Dizhoor AM, Pan ZH (2006) Ectopic expression of a microbial-type rhodopsin restores visual responses in mice with photoreceptor degeneration. Neuron 50:23-33.
Bittner AK, Diener-West M, Dagnelie G (2009) A survey of photopsias in self-reported retinitis pigmentosa. Retina 29:1513-1521.

Blankenship AG, Ford KJ, Johnson J, Seal RP, Edwards RH, Copenhagen DR, Feller MB (2009) Synaptic and extrasynaptic factors governing glutamatergic retinal waves. Neuron 62:230-241.

Bloomfield SA, Völgyi B (2009) The diverse functional roles and regulation of neuronal gap junctions in the retina. Nat Rev Neurosci 10:495-506.

Borowska JT, Trenholm S, Awatramani GB (2010) Intrinsic mechanisms in bipolar cells drive spontaneous network activity during retinal degeneration. ARVO Meet Abstr 51:2486.

Busskamp V, Duebel J, Balya D, Fradot M, Viney TJ, Siegert S, Groner AC, Cabuy E, Forster V, Seeliger M, Biel M, Humphries P, Paques M, Mohand-Said S, Trono D, Deisseroth K, Sahel JA, Picaud S, Roska B (2010) Genetic reactivation of cone photoreceptors restores visual responses in retinitis pigmentosa. Science 329:413-417.

Carlen PL, Skinner F, Zhang L, Naus C, Kushnir M, Perez Velazquez JL (2000) The role of gap junctions in seizures. Brain Res Rev 32:235-241.

Castro-Alamancos MA, Rigas P, Tawara-Hirata Y (2007) Resonance (similar to $10 \mathrm{~Hz}$ ) of excitatory networks in motor cortex: effects of voltagedependent ion channel blockers. J Physiol 578:173-191.

Chang B, Hawes NL, Hurd RE, Davisson MT, Nusinowitz S, Heckenlively JR (2002) Retinal degeneration mutants in the mouse. Vision Res 42:517-525.

Chen SJ, Mahadevappa M, Roizenblatt R, Weiland J, Humayun M (2006) Neural responses elicited by electrical stimulation of the retina. Trans Am Ophthalmol Soc 104:252-259.

Chua J, Fletcher EL, Kalloniatis M (2009) Functional remodeling of glutamate receptors by inner retinal neurons occurs from an early stage of retinal degeneration. J Comp Neurol 514:473-491.

Demas J, Eglen SJ, Wong RO (2003) Developmental loss of synchronous spontaneous activity in the mouse retina is independent of visual experience. J Neurosci 23:2851-2860.

Dräger UC, Hubel DH (1978) Studies of visual function and its decay in mice with hereditary retinal degeneration. J Comp Neurol 180:85-114.

Dräger UC, Olsen JF (1981) Ganglion-cell distribution in the retina of the mouse. Invest Ophthalmol Vis Sci 20:285-293.

Dudek FE, Yasumura T, Rash JE (1998) "Non-synaptic" mechanicsms in seizures and epileptogenesis. Cell Biol Int 22:793-805.

Eversmann B, Jenkner M, Hofmann F, Paulus C, Brederlow R, Holzapfl B, Fromherz P, Merz M, Brenner M, Schreiter M, Gabl R, Plehnert K, Steinhauser M, Eckstein G, Schmitt-Landsiedel D, Thewes R (2003) A $128 \times$ 128 CMOS biosensor array for extracellular recording of neural activity. IEEE J Solid State Circuits 38:2306-2317.

Fariss RN, Li ZY, Milam AH (2000) Abnormalities in rod photoreceptors, amacrine cells, and horizontal cells in human retinas with retinitis pigmentosa. Am J Ophthalmol 129:215-223.

Feigenspan A, Gustincich S, Bean BP, Raviola E (1998) Spontaneous activity of solitary dopaminergic cells of the retina. J Neurosci 18:6776-6789.

Feigenspan A, Teubner B, Willecke K, Weiler R (2001) Expression of neuronal connexin36 in AII amacrine cells of the mammalian retina. J Neurosci 21:230-239.

Fletcher EL (2000) Alterations in neurochemistry during retinal degeneration. Microsc Res Tech 50:89-102.

Flint AC, Connors BW (1996) Two types of network oscillations in neocortex mediated by distinct glutamate receptor subtypes and neuronal populations. J Neurophysiol 75:951-957.

Gao J, Schwartz G, Berry MJ 2nd, Holmes P (2009) An oscillatory circuit underlying the detection of disruptions in temporally-periodic patterns. Network 20:106-135.

Grafstein B, Murray M, Ingoglia NA (1972) Protein synthesis and axonal transport in retinal ganglion cells of mice lacking visual receptors. Brain Res 44:37-48.

Heckenlively JR, Yoser SL, Friedman LH, Oversier JJ (1988) Clinical findings and common symptoms in retinitis pigmentosa. Am J Ophthalmol 105:504-511.

Hombach S, Janssen-Bienhold U, Söhl G, Schubert T, Büssow H, Ott T, Weiler R, Willecke K (2004) Functional expression of connexin57 in horizontal cells of the mouse retina. Eur J Neurosci 19:2633-2640.

Hu EH, Bloomfield SA (2003) Gap junctional coupling underlies the shortlatency spike synchrony of retinal alpha ganglion cells. J Neurosci 23:6768-6777.

Humayun MS, Prince M, de Juan E Jr, Barron Y, Moskowitz M, Klock IB, 
Milam AH (1999) Morphometric analysis of the extramacular retina from postmortem eyes with retinitis pigmentosa. Invest Ophthalmol Vis Sci 40:143-148.

Kolb H, Gouras P (1974) Electron-microscopic observations of human retinitis pigmentosa, dominantly inherited. Invest Ophthalmol 13:487-498.

Kolomiets B, Dubus E, Simonutti M, Rosolen S, Sahel JA, Picaud S (2010) Late histological and functional changes in the $\mathrm{P} 23 \mathrm{H}$ rat retina after photoreceptor loss. Neurobiol Dis 38:47-58.

Lagali PS, Balya D, Awatramani GB, Münch TA, Kim DS, Busskamp V, Cepko CL, Roska B (2008) Light-activated channels targeted to ON bipolar cells restore visual function in retinal degeneration. Nat Neurosci 11:667-675.

Lambacher A, Jenkner M, Merz M, Eversmann B, Kaul RA, Hofmann F, Thewes R, Fromherz P (2004) Electrical imaging of neuronal activity by multi-transistor-array (MTA) recording at $7.8 \mu \mathrm{m}$ resolution. Appl Phys A Mater Sci Process 79:1607-1611.

Lambacher A, Vitzthum V, Zeitler R, Eickenscheidt M, Eversmann B, Thewes R, Fromherz P (2010) Identifying firing mammalian neurons in networks with high-resolution multi-transistor array (MTA). Appl Phys A Mater Sci Process 102:1-11.

Lin B, Koizumi A, Tanaka N, Panda S, Masland RH (2008) Restoration of visual function in retinal degeneration mice by ectopic expression of melanopsin. Proc Natl Acad Sci U S A 105:16009-16014.

Lin B, Masland RH, Strettoi E (2009) Remodeling of cone photoreceptor cells after rod degeneration in rd mice. Exp Eye Res 88:589-599.

Marc RE, Jones BW, Watt CB, Strettoi E (2003) Neural remodeling in retinal degeneration. Prog Retin Eye Res 22:607-655.

Margolis DJ, Detwiler PB (2011) Cellular origin of spontaneous ganglion cell spike activity in animal models of retinitis pigmentosa. J Ophthalmol pii:507037.

Margolis DJ, Newkirk G, Euler T, Detwiler PB (2008) Functional stability of retinal ganglion cells after degeneration-induced changes in synaptic input. J Neurosci 28:6526-6536.

Mastronarde DN (1989) Correlated firing of retinal ganglion cells. Trends Neurosci 12:75-80.

Mazzoni F, Novelli E, Strettoi E (2008) Retinal ganglion cells survive and maintain normal dendritic morphology in a mouse model of inherited photoreceptor degeneration. J Neurosci 28:14282-14292.

Mitzdorf U (1985) Current source-density method and application in cat cerebral cortex: investigation of evoked potentials and EEG phenomena. Physiol Rev 65:37-100.

Park SJ, Kim IB, Choi KR, Moon JI, Oh SJ, Chung JW, Chun MH (2001) Reorganization of horizontal cell processes in the developing FVB/N mouse retina. Cell Tissue Res 306:341-346.

Petit-Jacques J, Völgyi B, Rudy B, Bloomfield S (2005) Spontaneous oscillatory activity of starburst amacrine cells in the mouse retina. J Neurophysiol 94:1770-1780.

Pinto DJ, Patrick SL, Huang WC, Connors BW (2005) Initiation, propagation, and termination of epileptiform activity in rodent neocortex in vitro involve distinct mechanisms. J Neurosci 25:8131-8140.

Protti DA, Flores-Herr N, von Gersdorff H (2000) Light evokes $\mathrm{Ca}^{2+}$ spikes in the axon terminal of a retinal bipolar cell. Neuron 25:215-227.

Rufer M, Wirth SB, Hofer A, Dermietzel R, Pastor A, Kettenmann H, Unsicker K (1996) Regulation of connexin-43, GFAP, and FGF-2 is not accompanied by changes in astroglial coupling in MPTP-lesioned, FGF2-treated parkinsonian mice. J Neurosci Res 46:606-617.

Ryu SB, Ye JH, Goo YS, Kim CH, Kim KH (2010) Temporal response properties of retinal ganglion cells in rd1 mice evoked by amplitude-modulated electrical pulse trains. Invest Ophthalmol Vis Sci 51:6762-6769.

Santos A, Humayun MS, de Juan E Jr, Greenburg RJ, Marsh MJ, Klock IB, Milam AH (1997) Preservation of the inner retina in retinitis pigmentosa: a morphometric analysis. Arch Ophthalmol 115:511-515.

Sanyal S, Bal AK (1973) Comparative light and electron-microscopic study of retinal histogenesis in normal and rd mutant mice. Z Anat Entwicklungsgesch 142:219-238.

Sauvé Y, Girman SV, Wang S, Lawrence JM, Lund RD (2001) Progressive visual sensitivity loss in the Royal College of Surgeons rat: perimetric study in the superior colliculus. Neuroscience 103:51-63.

Sekirnjak C, Hulse C, Jepson LH, Hottowy P, Sher A, Dabrowski W, Litke AM, Chichilnisky EJ (2009) Loss of responses to visual but not electrical stimulation in ganglion cells of rats with severe photoreceptor degeneration. J Neurophysiol 102:3260-3269.
Sinha SR, Saggau P (2001) Imaging of 4-AP-induced, $\mathrm{GABA}_{\mathrm{A}}$-dependent spontaneous synchronized activity mediated by the hippocampal interneuron network. J Neurophysiol 86:381-391.

Söhl G, Maxeiner S, Willecke K (2005) Expression and functions of neuronal gap junctions. Nat Rev Neurosci 6:191-200.

Stasheff SF (2008) Emergence of sustained spontaneous hyperactivity and temporary preservation of OFF responses in ganglion cells of the retinal degeneration (rd1) mouse. J Neurophysiol 99:1408-1421.

Stone JL, Barlow WE, Humayun MS, de Juan E Jr, Milam AH (1992) Morphometric analysis of macular photoreceptors and ganglion cells in retinas with retinitis pigmentosa. Arch Ophthalmol 110:1634-1639.

Strettoi E, Pignatelli V (2000) Modifications of retinal neurons in a mouse model of retinitis pigmentosa. Proc Natl Acad Sci U S A 97:11020-11025.

Strettoi E, Raviola E, Dacheux RF (1992) Synaptic connections of the narrow-field, bistratified rod amacrine cell (AII) in the rabbit retina. J Comp Neurol 325:152-168.

Strettoi E, Porciatti V, Falsini B, Pignatelli V, Rossi C (2002) Morphological and functional abnormalities in the inner retina of the $\mathrm{rd} / \mathrm{rd}$ mouse. J Neurosci 22:5492-5504.

Strettoi E, Pignatelli V, Rossi C, Porciatti V, Falsini B (2003) Remodeling of second-order neurons in the retina of $\mathrm{rd} / \mathrm{rd}$ mutant mice. Vision Res 43:867-877.

Syed MM, Lee S, Zheng J, Zhou ZJ (2004) Stage-dependent dynamics and modulation of spontaneous waves in the developing rabbit retina. J Physiol 560:533-549.

Taketo M, Schroeder AC, Mobraaten LE, Gunning KB, Hanten G, Fox RR, Roderick TH, Stewart CL, Lilly F, Hansen CT, Overbeek PA (1991) FVB/N: an inbred mouse strain preferable for transgenic analyses. Proc Natl Acad Sci U S A 88:2065-2069.

Thoreson WB, Witkovsky P (1999) Glutamate receptors and circuits in the vertebrate retina. Prog Retin Eye Res 18:765-810.

Thyagarajan S, van Wyk M, Lehmann K, Löwel S, Feng G, Wässle H (2010) Visual function in mice with photoreceptor degeneration and transgenic expression of channelrhodopsin 2 in ganglion cells. J Neurosci 30:8745-8758.

Tian M, Jarsky T, Murphy GJ, Rieke F, Singer JH (2010) Voltage-gated Na channels in AII amacrine cells accelerate scotopic light responses mediated by the rod bipolar cell pathway. J Neurosci 30:4650-4659.

Traub RD, Jefferys JG, Miles R (1993) Analysis of the propagation of disinhibition-induced after-discharges along the guinea-pig hippocampal slice in vitro. J Physiol 472:267-287.

Uhhaas PJ, Singer W (2006) Neural synchrony in brain disorders: relevance for cognitive dysfunctions and pathophysiology. Neuron 52:155-168.

Vaithianathan T, Sagdullaev BT (2010) Functional remodeling of inner retinal synaptic transmission during photoreceptor degeneration. ARVO Meet Abstr 51:2483.

Veruki ML, Hartveit E (2002) All (rod) amacrine cells form a network of electrically coupled Interneurons in the mammalian retina. Neuron 33:935-946.

Veruki ML, Hartveit E (2009) Meclofenamic acid blocks electrical synapses of retinal AII amacrine and ON-cone bipolar cells. J Neurophysiol 101:2339-2347.

Veruki ML, Oltedal L, Hartveit E (2008) Electrical synapses between AII amacrine cells: dynamic range and functional consequences of variation in junctional conductance. J Neurophysiol 100:3305-3322.

Völgyi B, Chheda S, Bloomfield SA (2009) Tracer coupling patterns of the ganglion cell subtypes in the mouse retina. J Comp Neurol 512:664-687.

Wässle H (2004) Parallel processing in the mammalian retina. Nat Rev Neurosci 5:747-757.

Wong RO (1999) Retinal waves and visual system development. Annu Rev Neurosci 22:29-47.

Ye JH, Goo YS (2007) The slow wave component of retinal activity in $\mathrm{rd} / \mathrm{rd}$ mice recorded with a multi-electrode array. Physiol Meas 28:1079-1088.

Zeck GM, Masland RH (2007) Spike train signatures of retinal ganglion cell types. Eur J Neurosci 26:367-380.

Zrenner E, Bartz-Schmidt KU, Benav H, Besch D, Bruckmann A, Gabel VP, Gekeler F, Greppmaier U, Harscher A, Kibbel S, Koch J, Kusnyerik A, Peters T, Stingl K, Sachs H, Stett A, Szurman P, Wilhelm B, Wilke R (2010) Subretinal electronic chips allow blind patients to read letters and combine them to words. Proc Biol Sci. Advance online publication. Retrieved January 11, 2011. doi:10.1098/rspb.2010.1747. 